\title{
1 Parkin coordinates mitochondrial lipid remodeling to execute mitophagy
}

3 Chao-Chieh $\operatorname{Lin}^{1,3,7}$, Jin Yan $^{1,7}$, Meghan D. Kapur ${ }^{1,7}$, Kristi L. Norris ${ }^{1,7}$, Cheng-Wei Hsieh ${ }^{4}$,

4 Chun-Hsiang Lai ${ }^{1}$, Nicolas Vitale ${ }^{5}$, Kah-Leong Lim ${ }^{6}$, Ziqiang Guan ${ }^{2}$, Jen-Tsan Chi ${ }^{3}$, Wei-Yuan

5 Yang $^{4}$ and Tso-Pang Yao ${ }^{1 \#}$

6 Duke University Medical Center, Durham, NC, USA

7 1. Department of Pharmacology and Cancer Biology,

$8 \quad 2$. Department of Biochemistry,

9 3. Department of Molecular Genetics and Microbiology,

$10 \quad{ }^{4}$ Institute of Biological Chemistry, Academia Sinica, Taipei 115, Taiwan

115 . Institut des Neurosciences Cellulaires et IntégrativesUPR-3212 CNRS - Université de

12 Strasbourg

$13 \quad{ }^{6}$ Lee Kong Chian School of Medicine, Singapore

14 7. These authors contribute equally to this work.

$15 \quad{ }^{\#}$ Correspondence: yao00001@mc.duke.edu

16 Running Title: Mitochondrial lipid remodeling controls mitophagy

17 Key words: diacylglycerol, parkin, autophagy receptors, Golgi, Lipin-1, mitophagy 


\section{Abstract}

20 Mitochondrial failure caused by Parkin mutations contributes to Parkinson's disease. Parkin

21 binds, ubiquitinates, and targets impaired mitochondria for autophagic destruction. Robust

22 mitophagy involves peri-nuclear concentration of Parkin-tagged mitochondria, followed by

23 dissemination of juxtanuclear mitochondrial aggregates, and efficient sequestration of

24 individualized mitochondria by autophagosomes. Here, we report that the execution of complex

25 mitophagic events requires active mitochondrial lipid remodeling. Parkin recruits phospholipase

26 D2 to the depolarized mitochondria and generate phosphatidic acid (PA). Mitochondrial PA is

27 subsequently converted to diacylglycerol (DAG) by Lipin-1 phosphatase- a process that further

28 requires mitochondrial ubiquitination and ubiquitin-binding autophagic receptors, NDP52 and

29 Optineurin. We show that Optineurin transports, via Golgi-derived vesicles, a PA-binding factor

30 EndoB1 to ubiquitinated mitochondria, thereby facilitating DAG production. Mitochondrial

31 DAG activates both F-actin assembly to drive mitochondrial individualization, and

32 autophagosome biogenesis to efficiently restrict impaired mitochondria. Thus Parkin, autophagic

33 receptors and the Golgi complex orchestrate mitochondrial lipid remodeling to execute robust

34 mitophagy. 


\section{Introduction}

Mutations in the ubiquitin E3 ligase, Parkin (PARK2), are the most common cause of

42 early onset Parkinson's disease (AR-JP) ${ }^{1}$. Genetic and biochemical evidence have shown that

43 Parkin, working in conjunction with the mitochondrial kinase, PINK1 (PARK6), preserves a

44 healthy mitochondrial population ${ }^{2,3,4}$. Parkin and PINK1 maintain mitochondrial integrity, at

45 least in part, by recognizing and targeting the impaired and de-energized mitochondria to

46 autophagy- a double membrane vesicles that deliver mitochondria to lysosomes- for destruction ${ }^{5}$

$47{ }^{6}$. In a basic model, stabilized PINK1 recruits Parkin to depolarized mitochondria, where Parkin

48 catalyzes extensive ubiquitination to initiate mitophagy ${ }^{7,8,9}$. Mitochondrial ubiquitination serves

49 at least two main functions: marking mitochondrial outer membrane proteins for proteasome-

50 mediated degradation ${ }^{10,11}$; recruiting autophagosomes to sequester and deliver ubiquitin-tagged

51 mitochondria to lysosomes for degradation ${ }^{7,12}$. While the exact contribution of the proteasome

52 to mitophagy remains uncertain ${ }^{13}$, on the autophagy front, mitochondrial ubiquitination has been

53 shown to recruit multiple ubiquitin-binding autophagic receptors, including Optineurin (OPTN),

54 NDP52 and p62/SQSTM1 ${ }^{12,14,15}$, which bring together ubiquitinated mitochondria and

55 autophagosomes through their binding of LC3- a protein being conjugated to

56 phosphatidylethanolamine in the autophagosome membrane ${ }^{16}$. Detailed functional analyses,

57 however, have also revealed a more complex picture of this model. Whereas OPTN and NDP52

58 are required for mitophagy ${ }^{14,15}$, p62 appears to be dispensable in some studies but not others ${ }^{7,12,}$

5917,18 . Instead, p62 acts to coalesce ubiquitinated mitochondria into large aggregates at the

60 perinuclear region- a dramatic event yet without a clearly defined function ${ }^{17,18}$. Recent evidence 
61 further indicates that autophagosomes are actually synthesized "de novo" at or around

62 mitochondria tagged for destruction ${ }^{15,19,20}$. How Parkin and different autophagic receptors

63 coordinate local autophagosome biogenesis around condemned mitochondria to efficiently

64 execute mitophagy remains to be fully elucidated.

65 Intriguingly, when subject to massive uncoupling by CCCP, the large mitochondrial

66 populations tagged by Parkin are recognized and actively concentrated by microtubule-based

67 motors and form large perinuclear aggregates or inclusions ${ }^{12,21}$. Perinuclear mitochondrial

68 inclusions have also been observed in neurons from PD patients ${ }^{22,23}$, indicating that this process

69 is physiologically relevant. Juxtanuclear mitochondrial aggregates, or termed mito-aggresomes,

70 are subsequently dispersed into smaller units prior to their sequestration by autophagosomes

71 (mitophagosomes) and eventual degradation ${ }^{11}$. Electron microscopy has revealed that some

72 perinuclear mitochondria concentrated by Parkin and PINK1 are fused ${ }^{21}$. These findings imply

73 that dissemination of perinuclear mitochondrial aggregates would require active "de-

74 aggregation" and "fission" of mitochondria. Thus, the disposal of impaired mitochondria

75 involves elaborate temporal and spatial regulation orchestrated by Parkin. How Parkin

76 coordinates these events to achieve robust mitophagy is not well understood.

77 In this report, we present evidence that the recruitment of Parkin to depolarized

78 mitochondria initiates a series of lipid remodeling events, characterized by the sequential

79 production of mitochondrial phosphatidic acid (PA) and diacylglycerol (DAG). Using

80 fluorescent PA and DAG binding reporters, we present evidence that Parkin recruits a

81 phospholipase, PLD2, to the depolarized mitochondria to generate PA, while activates the PA 
82 phosphatase Lipin-1-dependent DAG production through mitochondrial ubiquitination that

83 recruits ubiquitin-binding autophagic receptors, OPTN and NDP52. We present evidence that

84 OPTN and NDP52, via Golgi-derived vesicles, deliver a lipid-remodeling factor EndoB1 to the

85 ubiquitinated mitochondria and stimulate DAG synthesis. Functionally, mitochondrial DAG

86 drives both the individualization of juxtanuclear mitochondrial aggregates by stimulating F-actin

87 assembly on mitochondria, and the production of autophagosomes, thereby coupling the

88 preparation of autophagible mitochondria units with autophagosome sequestration. Our findings

89 uncover local mitochondrial lipid remodeling as a novel and critical mechanism that ensures

90 robust mitophagy, and identify the Golgi complex as an important component of mitochondrial

91 QC machinery.

\section{Results:}

\section{Parkin stimulates mitochondrial PA and DAG accumulation}

94 PINK1 and Parkin have been shown to promote mitochondrial fusion in response to moderate

95 mitochondrial stresses induced by low-dose CCCP ${ }^{24}$. In addition to mitofusin (MFN)- and Drp1-

96 related GTPases, mitochondrial dynamics is affected by membrane lipid composition, in which

97 mitochondrial phosphatidic acid (PA) and diacylglycerol (DAG) stimulate fusion and fission

98 respectively ${ }^{25,26}$. We investigated whether mitochondrial PA or DAG is involved in Parkin-

99 mediated mitochondrial fusion. To this end, we employed specific fluorescent reporters that

100 bind PA (EGFP-Raf1-PABD, referred as PA reporter $\left.{ }^{27}\right)$ or DAG $(\mathrm{C} 1 \mathrm{~b} \delta-\mathrm{CFP} / \mathrm{YFP}$, referred as

101 YFP-DAGR) to monitor PA and DAG production ${ }^{26,28}$. However, when Parkin-expressing cells

102 were challenged by low-dose CCCP $(1 \mu \mathrm{M})$, no discernible change was observed for either the 
103 PA reporter or DAGR (not shown). Unexpectedly, when these cells were challenged by high-

104 dose CCCP $(10 \mu \mathrm{M})$ to activate global mitophagy, prominent accumulation of the PA-reporter on

105 Parkin-tagged mitochondria were observed, as early as 1 hour after CCCP treatment (Fig. 1a, 106 quantified in Fig. 1c). A different PA-binding reporter (Spo20p-GFP) ${ }^{29}$ showed similar

107 mitochondrial translocation in response to high-dose CCCP treatment (Supplementary Fig. 1a).

108 Mitophagy activated by antimycin/oligomycin treatment similarly induced concentration of the

109 PA reporter on mitochondria. (Supplementary Fig. 1b). Using mitochondria-targeted

110 photosensitizer KillerRed to damage a defined population of mitochondria ${ }^{20}$, the PA reporter was

111 again detected on Parkin-positive mitochondria (Supplementary Fig. 1d). Collectively, these

112 findings indicate that PA accumulates on impaired mitochondria tagged by Parkin.

113 To assess whether PA on Parkin-tagged mitochondria is further processed to DAG, we

114 employed a high-affinity DAG reporter (YFP-DAGR). Under basal conditions YFP-DAGR

115 mainly labeled perinuclear structures corresponding to the Golgi apparatus, which contains

116 abundant DAG ${ }^{30}$ (Fig. 1b, arrowhead). Upon mitophagy activation by either CCCP or

117 antimycin/oligomycin treatment, dramatic recruitment of YFP-DAGR to Parkin-tagged

118 mitochondria was observed (Fig. 1b-1c and Supplementary Fig. 1c). Neither YFP-DAGR nor the

119 PA reporter accumulated on mitochondria in the absence of Parkin (not shown); however, both

120 reporters were detected on mitochondria targeted for mitophagy in SH-SY5Y cells, which

121 express endogenous Parkin (Supplementary Fig. 1e-f). Altogether, the lipid reporter assays

122 indicate that Parkin stimulates PA and DAG production on mitochondria tagged for autophagy.

\section{Mitochondrial DAG production requires Parkin E3 ligase activity}


124 Parkin activates mitophagy by catalyzing mitochondrial ubiquitination. We assessed two AR-JP

125 associated Parkin mutants, T240R and T415N, which are deficient in the ubiquitin E3 ligase

126 activity and cannot support mitophagy ${ }^{12}$. As shown in Fig. 1d, neither mutations affected the

127 mitochondrial translocation of the PA-reporter. In contrast, mitochondrial YFP-DAGR

128 accumulation was strongly inhibited (Fig. 1e, quantified in 1f). Thus, mitochondrial

129 ubiquitination is required for mitochondrial DAG, but not PA, production. These findings

130 suggest that mitochondrial PA production is initiated by the recruitment of Parkin while DAG

131 production further requires Parkin E3 ligase activity.

\section{PLD2 and Lipin-1 catalyze sequential production of mitochondrial PA and DAG}

133 We next determined how Parkin orchestrates mitochondrial lipid remodeling. Phospholipase D is

134 the primary enzyme that produces PA. A mitochondrial resident phospholipase D, Mito-PLD

135 (PLD6), generates mitochondrial PA by hydrolyzing cardiolipin ${ }^{25}$. However, PA reporter

136 accumulated normally on Parkin-tagged mitochondria in Mito-PLD knockout fibroblasts in

137 response to CCCP treatment (data not shown). We next investigated whether Parkin recruits a

138 cytosolic PLD to depolarized mitochondria and catalyze PA production. Of two widely

139 expressed PLD members, we found that PLD2 (Fig. 2a), but not PLD1 (Supplementary Fig. 2a),

140 translocated onto Parkin-tagged mitochondria upon treatment with CCCP or

141 oligomycin/antimycin (Supplementary Fig. 2b). Immunoblotting confirmed that endogenous

142 PLD2, along with Parkin, became enriched in the mitochondrial fraction in response to CCCP

143 treatment (Fig. 2b). To determine the role of PLD2 on mitochondrial PA production, we

144 inhibited PLD2 by a specific inhibitor, VU $0364739^{31}$, or by siRNA-mediated knockdown. 
145 Importantly, mitochondrial accumulation of the PA reporter was markedly suppressed by either

146 pharmacological inhibition (Fig. 2c-d) or siRNA-mediated knockdown of PLD2 (Supplementary

147 Fig. 2d-e). PLD2 inhibition also markedly prevented mitochondrial YFP-DAGR accumulation

148 (Supplementary Fig. 2f). These findings indicate that Parkin stimulates mitochondrial PA

149 production by recruiting PLD2, and that PLD2-generated PA is a precursor to mitochondrial

150 DAG.

151 To determine how PA is converted to DAG, we knocked down Lipin-1, a major cytosolic

152 phosphatase that dephosphorylates PA to DAG ${ }^{26,32}$. Lipin-1 knockdown led to a dramatic loss

153 of DAGR-YFP on Parkin-tagged mitochondria (Fig. 2e, Lipin-1 KD) while the PA reporter was

154 not affected (Supplementary Fig. 2h). Re-expression of a siRNA-resistant WT Lipin-1 in Lipin-1

155 KD cells significantly restored mitochondrial DAGR-YFP while a catalytic inactive mutant

156 (Lipin-1 CD) was ineffective (Fig. 2f). Together, these findings demonstrate that PLD2 and

157 Lipin-1 work in tandem to generate PA and DAG on mitochondria tagged by Parkin.

158 DAG coordinates F-actin-dependent mitochondrial dissemination and mitophagy

159 Compared to WT control, we found that Lipin-1 knockdown cells were less efficient in 160 mitochondrial clearance, as shown by the significant retention of mitochondria following CCCP

161 treatment (Fig. 3a, bottom panels, quantified in Fig. 3b). Immunoblotting analysis confirmed that 162 autophagy-dependent degradation of multiple mitochondrial markers was impaired in lipin-1 KD 163 cells, whereas proteasome-mediated degradation of MFN1 was unaffected (Fig. 3c and 164 Supplementary Fig. 2i). PLD2 inhibition, which suppressed the production of mitochondrial PA, 165 the precursor to DAG, also reduced the efficiency of mitophagy (Supplementary Fig. 2j). These 
166 results support the conclusion that mitochondrial DAG production is important for Parkin167 mediated mitophagy.

168 During mitophagy, individual mitochondria became dispersed from the perinuclear 169 aggregates prior to their sequestration by autophagosomes ${ }^{11}$. Indeed, we observed many small 170 Parkin-positive mitochondria separated from the perinuclear cluster during mitophagy (Fig. 3d, 171 Top Panels). Strikingly, in Lipin-1 KD cells, significantly fewer "individualized and dispersed" 172 mitochondria were observed. Instead, mitochondria frequently remained as large perinuclear 173 aggregates (Fig. 3d, Bottom Panels; quantified in Fig. 3e). These findings indicate that Lipin-1 is 174 required for the dispersion of mitochondrial aggregates prior to autophagic sequestration.

175 Autophagic clearance of perinuclear protein inclusions, the aggresomes, requires active de176 aggregation implemented by the actin cytoskeleton ${ }^{33}$. Because DAG is a known activator of F177 actin assembly on the plasma membranes and endosomes ${ }^{34}$, 35, we asked whether Lipin-1178 dependent DAG production activates mitochondrial de-aggregation by promoting F-actin 179 assembly. To this end, we first used an F-actin binding probe, RFP-LifeAct ${ }^{36}$, to assess if F-actin 180 was assembled around mitochondrial aggregates. As shown in Fig. 3f, prominent LifeAct181 positive signals were detected on de-aggregated mitochondria (Middle Panels), suggesting that 182 F-actin is involved in mitochondrial de-aggregation. Supporting this hypothesis, inhibition of F183 actin assembly by cytochalasin B (Cyto B) markedly suppressed the appearance of RFP-LifeAct 184 positive mitochondria (Fig. 3f, Bottom Panels) and the dispersion of mitochondrial aggregates 185 (Quantified in Fig. 3 g). Importantly, Lipin-1 knockdown also suppressed the appearance of 186 LifeAct positive-mitochondria (Fig. 3h). These results support the conclusion that Lipin-1- 
187 dependent production of mitochondrial DAG stimulates local F-actin assembly to divide and

188 disperse perinuclear mitochondrial aggregates for autophagic degradation.

\section{Lipin1-dependent DAG stimulates autophagosome production}

We noticed that abundant accumulation of YFP-DAGR on mitochondria apparently retarded

191 mitochondrial clearance, as indicated by the continuous accumulation of YFP-DAGR positive

192 mitochondria after prolonged CCCP treatment (Fig. 1c, $18 \mathrm{hr}$ ). This finding is consistent with the

193 notion that high affinity DAGR can mask mitochondrial DAG from the downstream effectors.

194 To follow the fate of DAG at a later stage of mitophagy, we employed another DAG reporter

195 with a lower binding affinity, C1(2)-mRFP (referred as RFP-DAGR ${ }^{37}$ ). Under basal condition,

196 RFP-DAGR also mainly labeled the Golgi complex (Fig. 4a, top panels, arrowhead) positive for

197 a trans-Golgi marker TGN46 (Supplementary Fig. 3a). Interestingly, upon CCCP treatment,

198 many RFP-DAGR positive vesicles encompassing dispersed parkin-tagged mitochondria were

199 observed (Fig. 4b, Top Panels). Similar structures were also detected using the YFP-DAGR

200 reporter expressed at a lower concentration (Supplementary Fig. 3b). Importantly, RFP-DAGR -

201 labeled vesicles are frequently positive for the autophagosome marker LC3 (Fig. 4b) suggesting

202 that mitochondrial DAG is also involved in the production of autophagosomes that restrict

203 damaged mitochondria (i.e. mitophagosomes). Supporting this possibility, the abundance of

204 LC3-positive vesicles was markedly reduced in Lipin-1 KD cells after CCCP treatment (Fig. 4b,

205 bottom panels, and quantified in Fig. 4c). The LC3 signals remained in Lipin-1 KD cells were

206 typically small and did not contain mitochondria (Fig. 4b, Zoomed Insets). As expected,

207 mitophagy-associated RFP-DAGR induction was suppressed in Lipin-1 KD (Fig. 4b, bottom 
panels, Red). Collectively, these findings indicate that Lipin-1 and mitochondrial DAG

209 production stimulate mitophagosome production.

210 To confirm the role of DAG in mitophagosome production, we supplied Lipin-1 KD cells

211 with a cell permeable 1,2-dipalmitoyl-sn-glycerol (DPG), an effector DAG species produced by

212 Lipin-1 ${ }^{38}$. DPG supplement (+DPG Panels) markedly stimulated both the appearance of

213 dispersed mitochondria and large LC3-positive vesicles, many of those contained mitochondria

214 (Fig. 4d, Zoomed Inset; Quantified in Fig. 4e-f). These results indicate that mitochondrial DAG

215 produced by Lipin-1 promotes both mitochondrial dissemination and the production of

216 autophagosomes that sequester dispersed mitochondria.

\section{Mitochondrial DAG production requires ubiquitin-binding NDP52 and Optineurin}

218 Parkin-dependent mitochondrial DAG production requires its ubiquitin E3 ligase activity (Fig.

219 1e). Mitochondrial ubiquitination was shown to recruit ubiquitin-binding autophagic receptors

220 OPTN and NDP52 $2^{14,15}$. We, therefore, determined if OPTN and NDP52 are involved in

221 mitochondrial DAG production. Consistent with their redundant functions in mitophagy ${ }^{15}$,

222 individual knockdown of OPTN or NDP52 had no discernable effect on mitochondrial YFP-

223 DAGR accumulation (Supplemental Fig. 4a). Simultaneous knockdown of these autophagic

224 receptors, however, abrogated mitochondrial YFP-DAGR accumulation (Fig. 5a-b). Re225 expression of an siRNA-resistant WT OPTN, but not a ubiquitin-binding deficient (E478G) 226 mutant OPTN ${ }^{14}$, restored mitochondrial DAGR recruitment, supporting a specific and ubiquitin227 dependent activity of OPTN in mitochondrial lipid remodeling (Fig. 5b). Consistent with their 228 role in mitochondrial DAG production, OPTN/NDP52 KD also suppressed the formation of 
RFP-DAGR-positive LC3 vesicles (mitophagosomes, Supplementary Fig. 4b). Importantly, exogenous DPG treatment markedly increased mitophagy in OPTN/NDP52 KD cells (Fig. 5c-d).

231 These findings show that a key function of OPTN and NDP52 in mitophagy is to promote

232 mitochondrial DAG production.

\section{Mitochondrial DAG production requires Golgi associated EndoB1}

234 Although commonly known as autophagic receptors, OPTN and NDP52 were initially

235 characterized as trans-Golgi associated proteins that regulate Golgi vesicle transport ${ }^{39,40}$. Indeed,

236 fluorescence-tagged OPTN (OPTN-Cherry) appeared as perinuclear vesicle-like structures that

237 were partially co-localized with a trans-Golgi marker, TGN38 (TGN38-GFP) (Fig. 6a, Top

238 Panels). Importantly, TGN38, along with OPTN, became accumulated on Parkin-tagged

239 mitochondria upon CCCP treatment (Fig. 6a, Bottom Panels). These observations suggest that

240 OPTN-TGN38-associated vesicles translocate onto ubiquitinated mitochondria tagged by Parkin.

241 These findings raise the possibility that OPTN might deliver factor(s) from the Golgi complex or

242 associated vesicles to ubiquitinated mitochondria, and thereby stimulate Lipin1-dependent DAG

243 production. We searched for potential Golgi factor(s) that is known to modify membrane lipid

244 composition and regulate mitophagy. One such a candidate identified is Endophilin B1 (EndoB1,

245 also known as Bif-1), which was reported to possess an intrinsic PA-binding activity ${ }^{41}$ and is

246 require for mitophagy ${ }^{41}, 42$. We found that EndoB1 was concentrated on the OPTN-positive

247 vesicles under basal conditions. In response to CCCP, EndoB1 became associated with Parkin-

248 and OPTN-tagged mitochondria (Fig. 6b). Mitochondrial recruitment of EndoB1, along with

249 OPTN, was confirmed by immunoblotting (Fig. 6c). Supporting EndoB1 as a potential cargo 
250 delivered to mitochondria by OPTN/NDP52, mitochondrial recruitment of EndoB1 was

251 markedly reduced in OPTN/NDP52 KD cells (Fig. 6d-e). Importantly, knockdown of EndoB1

252 suppressed the accumulation of YFP-DAGR on Parkin-tagged mitochondria (Fig. 6f-g) but had

253 no effect on PA reporter translocation (Supplementary Fig. 5a). Consistent with its effect on

254 mitochondrial DAG production, EndoB1 knockdown also reduced the production of DAGR-

255 positive LC3 vesicles (Supplementary Fig. 5b), These findings indicate that OPTN and NDP52

256 promote mitochondrial DAG production and mitophagy, at least in part, by delivering EndoB1 to

257 ubiquitinated mitochondria.

\section{Discussion}

259 Large-scale mitophagy activated by Parkin and CCCP treatment involves elaborate steps

260 including tagging and transporting impaired mitochondria to the MTOC, followed by active

261 dispersion to generate autophagible mitochondrial units, and de novo assembly of

262 autophagosomes to restrict the dispersed mitochondria. In this report, we presented evidence that

263 Parkin coordinates these complex events, at least in part, by stimulating local lipid remodeling on

264 impaired mitochondria. The sequential production of mitochondrial PA and DAG activates actin-

265 dependent dispersion of aggregated mitochondria and local autophagosome production, thereby

266 enabling the efficient disposal of a large population of impaired mitochondria.

267 Our data show that Parkin orchestrates mitochondrial lipid remodeling via two enzymes, PLD2

268 and Lipin-1. Co-immunoprecipitation analysis indicates that Parkin can associate with PLD2 in

269 response to mitophagy activation (Supplementary Fig. 2c). We, therefore, propose that the

270 production of PA entails stable mitochondrial interaction of Parkin, which recruits PLD2 to the 
271 impaired mitochondria. The subsequent conversion of PA to DAG further requires Parkin

272 ubiquitin ligase activity, indicating that Lipin-1, by itself, is not able to efficiently convert

273 mitochondrial PA to DAG. Unlike PLD2, we did not detect apparent recruitment of Lipin-1 to

274 Parkin-tagged mitochondria (data not shown). Instead, our data suggest that ubiquitin-binding

275 autophagy receptors, OPTN and NDP52, deliver one or more co-factors to ubiquitinated

276 mitochondria to enable Lipin-1-dependent DAG production. One such a co-factor we identified

277 is EndoB1, which has an intrinsic PA binding activity ${ }^{41}$. Our evidence suggests that EndoB1 is

278 delivered by OPTN-positive Golgi-vesicles to ubiquitinated mitochondria (Fig. 6), where the

279 mitochondrion-localized EndoB1 might bridge Lipin-1 and mitochondrial PA to produce DAG.

280 Therefore, Parkin utilizes both ubiquitin-independent and -dependent process to orchestrate

281 mitochondrial lipid remodeling.

282 The analysis of mitophagy phenotypes in Lipin-1-deficient cells has uncovered two inter-

283 connected activities for mitochondrial DAG: activation of F-actin assembly and production of

284 autophagosomes. It was previously observed that dispersion of perinuclear mitochondrial

285 aggregates precedes autophagic sequestration of impaired mitochondria ${ }^{11}$. Our data now provide

286 evidence that the dispersion of mitochondrial aggregate is facilitated by the assembly of the actin

287 cytoskeleton surrounding "aggregated" mitochondria (Fig. 3f, Middle Panels). Either inactivation

288 of Lipin-1 or treatment with an actin polymerization inhibitor, cytochalasin B, effectively

289 suppress mitochondrial de-aggregation (Fig. 3f, Bottom Panels and Fig. 3h). The actin

290 cytoskeleton could, in principle, enable mechanical force production to separate individual

291 mitochondria from the perinuclear cluster- a scenario similar to the actinomyosin-dependent de- 
292 aggregation of perinuclear protein inclusions, the aggresomes ${ }^{33}$. Indeed, cortactin, a key co293 factor for F-actin assembly, is required for the clearance of both aggresomes and mitochondrial 294 aggregates ${ }^{12,43}$. Evidence also showed that active mitochondrial division mediated by Drp1 is 295 necessary for efficient mitophagy ${ }^{10}$. Drp1-mediated mitochondrial fission also requires the 296 cortactin-dependent actin cytoskeleton and involves EndoB1 ${ }^{44,45,46}$. These findings support a 297 model whereby mitochondrial DAG produced by EndoB1 and Lipin-1 nucleates local F-actin 298 assembly to enable mitochondrial fission and dispersion.

299 Our analysis also revealed that Parkin-dependent DAG promotes mitophagosome 300 production (Fig. 4). Accordingly, local production of DAG on Parkin- and ubiquitin-tagged 301 mitochondria could, in principle, simultaneously activate focal mitochondrial fission/dispersion 302 and de novo autophagosome production - providing a coupling mechanism to ensure 303 "condemned" mitochondria are efficiently "restricted" by autophagosomes. Supporting the 304 instructive role of DAG in autophagy activation, a cell-permeable DAG (DPG) can stimulate 305 LC3-positive vesicle production in Lipin-1 KD cells (Fig. 4d-f). DPG treatment also markedly 306 restored mitophagy in OPTN/NDP52 KD cells (Fig. 5c-d). The finding revealed that one key 307 function of autophagic receptors in mitophagy is to stimulate DAG production. Interestingly, 308 recent studies have revealed a surprising role of the actin cytoskeleton on autophagosome 309 formation 47,48 . We propose that DAG-mediated F-actin assembly coordinates both the 310 dispersion of mitochondria and local autophagosome assembly. Our data, however, do not 311 exclude the potential effect of mitochondrial DAG on proteasome activity during mitophagy. 
How mitochondrial DAG activates actin assembly remains to be determined. By mass

313 spectrometry-based lipid analysis, we have detected a dramatic accumulation ( $>10$ fold) of

314 selected mitochondrial DAG species on mitochondria tagged for autophagy in response to CCCP

315 treatment (Supplementary Fig. 6). This finding not only confirmed the production of

316 mitochondrial DAG during mitophagy but also revealed that most prominently induced DAG

317 species were those with long acyl chains (C38:4 and C38:3 as well as 36:2 and 36:1) while C32

318 and C34 species were not induced (Supplementary Fig. 6). Both C38:4 DAG (1-stearoyl-2-

319 arachidonoyl-sn-glycerol; SAG) and C36:2 (1-stearoyl-2-linoleoyl-sn-glycerol; SLG) are potent

320 PKC activators. These findings raise the possibility that mitochondrial DAG plays a signaling

321 role and locally activates PKC on Parkin-tagged mitochondria to affect mitophagy. We further

322 note that many LC3-positive autophagosomes produced during mitophagy are also labeled by the

323 DAGR (Fig. 4b, Supplementary Fig. 3b), suggesting that DAG might have additional functions

324 in the later stage of mitophagy. Future studies will be required to test these possibilities.

325 Perinuclear mitochondrial inclusions have been observed in both PD patients and a mouse PD

326 model $^{22,23,49}$. Perinuclear protein inclusion, the Lewy body, is a defining pathological feature of

$327 \mathrm{PD}^{50}$. Despite their prevalence, the physiological purpose of concentrating "cellular junk" to the

328 perinuclear region remains uncertain. Our findings suggest that perinuclear OPTN-associated

329 and Golgi-derived vesicles are involved in mitochondrial DAG production and mitophagy. As

330 the Golgi complex is normally located at the MTOC/perinuclear region, retrograde transport of

331 impaired mitochondria and protein aggregates would place them close to the Golgi complex or

332 Golgi-derived vesicles. Such an arrangement, in principle, could ensure a ready access of 
333 damaged mitochondria or protein aggregates to the OPTN/NDP52 vesicles for autophagic

334 processing. Interestingly, the endoplasmic reticulum (ER) was proposed to play a critical role in

335 mitophagy initiated at focally damaged mitochondria ${ }^{20}$. We surmise that when the production of

336 damaged mitochondria exceeds the capacity of local autophagic degradation machinery

337 (peripheral autophagy)- for example under chronic pathological conditions associated with

338 neurodegenerative disease- the "overflowed" ubiquitin- and p62-tagged mitochondria would

339 become engaged and transported by dynein motors to access the Golgi-associated vesicles and

340 perinuclear autophagy machinery (central autophagy). Accordingly, the prevalence of

341 perinuclear inclusions in PD patients could reflect the active role of the Golgi complex/vesicles

342 in managing excessive cellular junk that accumulates in ailing neurons. Interestingly, many PD-

343 associated PARK genes have been linked to Golgi integrity or Golgi-associated vesicular

344 trafficking $51,52,53,54,55,56$. It is tantalizing to speculate that Golgi dysfunction might be a risk

345 factor for PD and that impaired central autophagy would weaken the clearance of protein

346 aggregates or mitochondria, resulting in perinuclear inclusions.

347 In conclusion, our study has uncovered PLD2-EndoB1-Lipin 1 dependent mitochondrial

348 lipid remodeling as a critical event in Parkin-dependent autophagic clearance of impaired

349 mitochondria. Indeed, aberrant accumulation of mitochondria has been noted in Lipin 1 and

350 EndoB1 knockout mice ${ }^{38,57}$, although the state of mitochondria in PLD2 knockout mice remains

351 to be characterized. The effect of exogenous DPG to substitute for endogenous DAG in

352 stimulating autophagosome production and mitophagy suggests that specific DAG-mimetics

353 might improve mitochondrial QC in ailing neurons. Similar to impaired mitochondria, we have 
354 found that photo-damaged lysosomes targeted for autophagic clearance ${ }^{58}$ also accumulated the 355 PA reporter (W.Y.Y, Unpublished observation). An association of DAG on salmonella targeted 356 for xenophagy has similarly been reported ${ }^{59}$. Thus, our study suggests that local lipid remodeling 357 might be a conserved mechanism to implement autophagy-dependent organelle quality control 358 (QC) and antibacterial defense. 


\section{Antibodies and Reagents}

365 The following primary antibodies were used: anti-Tom20 (Santa Cruz sc-11415), anti366 cytochrome c (BD Bioscience 556432), anti-GAPDH (Cell Signaling 14C10, 2118), anti-actin

367 (Sigma AC-15, A1978), anti-EndoB1 (R\&D, AF7456), anti-Tim23 (BD Bioscience), anti-Mfn2

368 (Santa Cruz sc-50331), anti-Mfn1 (Santa Cruz), anti-LC3 (MBL International), anti-FLAG (M2

369 Sigma F7425), anti-lipin-1 (Cell Signaling 5195), anti-PLD2 (Scbt sc-515744), Citrate

370 synthetase (GTX110624, GeneTex), Hsp60 (Cell Signaling, 4870). Secondary antibodies were

371 from Jackson Immunochem or Invitrogen.

372 The following reagents were used: DMSO (Sigma D8418), carbonyl cyanide 5-chloro-

373 phenylhydrazone (CCCP) (Sigma), PLD2 inhibitor VU 0364739 hydrochloride (Tocris 4171),

374 Cytochalasin B (Sigma), and 1,2-dipalmitoyl-sn-glycerol (Sigma D9135)

\section{Cell Culture}

376 Hela and HEK293T cells were obtained from Duke Cell Culture Facility (Durham, NC, USA).

377 The cells were cultured in Dulbecco's modified Eagle's medium (DMEM; GIBCO-11995)

378 supplemented with $10 \%$ fetal bovine serum and $1 \times$ antibiotics (penicillin, 10,000 UI/ml and

379 streptomycin, 10,000 UI/ml). These cell lines have been authenticated by STR DNA profiling

380 and validated to be mycoplasma-free and before being frozen by the Duke Cell Culture Facility

381 (Durham, NC, USA). All cells were maintained at $37^{\circ} \mathrm{C}$ and $5 \% \mathrm{CO}_{2}$.

\section{$382 \quad$ Plasmids and Transfection}


383 The following plasmids/siRNAs/shRNAs were used: GFP-Parkin and mutants as previously 384 described ${ }^{12}$; mcherry-Parkin ${ }^{20}$; GFP-Raf1-PABD and CFP/YFP-DAGR ${ }^{26}$; mcherry-optineurin $^{14}$;

385 YFP-PLD1 and YFP-PLD2 (Gifts from W.G. Zhang); RFP-LifeAct (ibidi). mCherry-Raf1386 PABD was generated by sub-cloning the PABD domain from GFP-Raf1-PABD to 387 mCherry2(C1). Expression plasmids for WT and catalytic mutant mouse Lipin-1 were obtained 388 from Addgene. Lipin-1 siRNA 5'-GAAUGGAAUGCCAGCUGAA-3' and 3'389 UUCAGCUGGCAUUCCAUUC-5' (Invitrogen; HSS118307 (Sigma); optineurin siRNA 390 (Invitrogen 4392420), NDP52 siRNA 5'-UUCAGUUGAAGCAGCUCUGUCUCCC-3,60, 391 EndoB1 5iRNA 5'-UGUUUAUACGACUUGGAGCUU-3' ${ }^{\prime} \quad$ and '392 AAGCUCCAAGUCGUAUAAACA-5' (Invitrogen), control siRNA (Ambion). Expression 393 plasmids were transfected in HeLa and YFP-Parkin HeLa using Xtreme Gene 9 (Roche) 394 according to manufacturer's directions. Cells were transfected and treated 24-48 hours later. 395 shRNA plasmids were transfected as stated above, but cells were not treated until 3-5 days later. 396 siRNAs were transfected using RNAi MAX (Invitrogen) according to the manufacturer's 397 directions and cells treated 48-72 hours later.

\section{Immunofluorescence microscopy and quantification}

399 Cells were seeded on coverslips, transfected, treated as indicated, and fixed in 4\% PFA for 15 400 minutes. Coverslips were rinsed in PBS, permeabilized with $0.2 \%$ Triton-X 100 in PBS for 5 401 minutes, blocked in 10\% BSA for 20 minutes in a humidified chamber, and incubated in primary 402 antibodies diluted in 10\% BSA overnight at 4C, followed by secondary antibodies in $10 \%$ BSA 403 for 30 minutes. Coverslips were mounted on slides using Fluromount G (Southern Biotech). 
404 Slides were analyzed on a Leica SP5 confocal microscope using 100x/1.4-0.70NA or 40x oil

405 objective (Leica Plan Apochromat). Z-stack images were acquired using the Leica LAS AF

406 program software and maximum projections used in analyses and figures. Changes to brightness

407 and contrast were performed in ImageJ. Number and size of vesicles were measured and 408 quantified using Particle Analysis module in Image J.

409 For all immunofluorescent quantifications, at least 35 to 50 cells were counted for 2-3 separate 410 experiments. Graphs represent means +/- SEM. For comparison of two conditions, a two-tailed,

411 unequal student's t-test was performed with $\mathrm{p}<0.05$ considered significant. For all other 412 comparisons, a one-way or two-way ANOVA was conducted.

\section{Preparation of mitochondrial fractions}

414 The isolation of mitochondria from cultured cells was performed by following manufacturer's 415 protocol (\#89874, ThermoFisher). In brief, after CCCP treatment, the cells harvested from a 10

$416 \mathrm{~cm}$ plate $\left(\sim 3 \times 10^{4}\right.$ cells $)$ were first resuspended in reagent A. Cell membrane was then lysed 417 using a reagent-based method by adding reagent B. After removing cell debris by low-speed 418 centrifugation, the mitochondrial fractions were collected in pellets with high speed 419 centrifugation. The remaining supernatant is cytosol fraction. Mitochondrial fractions were 420 further subject to Western blots or lipidomic analysis.

\section{$421 \quad$ Western blots}

422 HeLa or YFP-Parkin HeLa were seeded onto $10 \mathrm{~cm}$ or $6 \mathrm{~cm}$ dishes, transfected as outlined above, 423 and treated as described. For western blot analysis, whole cell lysates were collected using $424170 \mathrm{mM}$ NETN buffer, incubated at $4^{\circ} \mathrm{C}$ for 30 minutes, spun, supernatant collected, and protein 
425 concentration measured using the BCA assay (Thermo Scientific). Samples were normalized,

426 diluted in XT buffer with reducing agent (BioRad), and boiled for 5 minutes at $100^{\circ} \mathrm{C}$. $4-20 \%$

427 TGX gels (BioRad) were used, followed by transfer to nitrocellulose membranes. Primary 428 antibodies were added to $2 \% \mathrm{BSA}$, incubated overnight at $4 \mathrm{C}$, followed by rinsing, secondary 429 antibody incubation for 1 hour at room temperature, rinsing, and developing using either ECL

430 PicoWest (Thermo Scientific) or ECL Pro Lightning (Perkin-Elmer). Films were scanned, 431 cropped, and adjusted for brightness and contrast in Photoshop. Density of bands was measured

432 using Image J.

\section{Lipid extraction and LC/MS analysis}

434 Lipid extraction of purified mitochondria was performed using a modified Bligh-Dyer method as 435 previously decried ${ }^{61}$. For LC/MS analysis, the dried lipid extracts were dissolved in 436 chloroform/methanol $(2: 1, \mathrm{v} / \mathrm{v})$ and injected for normal phase LC/MS analysis on an Agilent 4371200 Quaternary LC system equipped with an Ascentis Silica HPLC column (5 $\mu \mathrm{m}, 25 \mathrm{~cm} \times 2.1$ $438 \mathrm{~mm}$, Sigma-Aldrich, St. Louis, MO). Mobile phase A consisted of 439 chloroform/methanol/aqueous ammonium hydroxide (800:195:5, v/v/v); mobile phase B 440 consisted of; mobile phase C consisted of. The elution program consisted of the following: 100\% 441 mobile phase A (chloroform/methanol/aqueous ammonium hydroxide; 800:195:5, v/v/v) was 442 held isocratically for $2 \mathrm{~min}$ and then linearly increased to $100 \%$ mobile phase B 443 (chloroform/methanol/water/aqueous ammonium hydroxide; 600:340:50:5, v/v/v/v) over $14 \min$ 444 and held at $100 \% \mathrm{~B}$ for $11 \mathrm{~min}$. The LC gradient was then changed to $100 \%$ mobile phase C 445 (chloroform/methanol/water/aqueous ammonium hydroxide; 450:450:95:5, v/v/v/v) over 3 min 
446 and held at $100 \% \mathrm{C}$ for $3 \mathrm{~min}$, and finally returned to $100 \%$ A over $0.5 \mathrm{~min}$ and held at $100 \% \mathrm{~A}$

447 for $5 \mathrm{~min}$. The LC eluent (with a total flow rate of $300 \mu \mathrm{l} / \mathrm{min}$ ) was introduced into the ESI

448 source of a high resolution TripleTOF5600 mass spectrometer (Sciex, Framingham, MA).

449 Instrumental settings for negative ion ESI/MS analysis of lipid species were as follows: IS= -

$4504500 \mathrm{~V} ; \mathrm{CUR}=20 \mathrm{psi} ; \mathrm{GSI}=20 \mathrm{psi} ; \mathrm{DP}=-55 \mathrm{~V}$; and $\mathrm{FP}=-150 \mathrm{~V}$. The MS/MS analysis used

451 nitrogen as the collision gas. Data analysis was performed using Analyst TF1.5 software (Sciex,

452 Framingham, MA).

453 Data availability

454 All data supporting the findings of this study are available from the authors upon reasonable 455 request. The source data underlying Figs 2d, 2f, 3b, 4e ,4f ,5b and 5d and Supplementary Figs 1a 456 and $5 \mathrm{~d}$ are provided as a Source Data file. Uncropped images of Western blots are shown 457 (Supplementary Fig. 7). 


\section{References}

1. Shimura $\mathrm{H}$, et al. Familial Parkinson disease gene product, parkin, is a ubiquitin-protein

9. Narendra DP, et al. PINK1 is selectively stabilized on impaired mitochondria to activate

2. Park J, et al. Mitochondrial dysfunction in Drosophila PINK1 mutants is complemented by parkin. Nature 441, 1157-1161 (2006).

3. Clark IE, et al. Drosophila pink1 is required for mitochondrial function and interacts genetically with parkin. Nature 441, 1162-1166 (2006).

4. Yang Y, et al. Mitochondrial pathology and muscle and dopaminergic neuron degeneration caused by inactivation of Drosophila Pink1 is rescued by Parkin. Proc Natl Acad Sci U S A 103, 10793-10798 (2006).

5. Narendra D, Tanaka A, Suen DF, Youle RJ. Parkin is recruited selectively to impaired mitochondria and promotes their autophagy. J Cell Biol 183, 795-803 (2008).

6. Zhang CW, Hang L, Yao TP, Lim KL. Parkin Regulation and Neurodegenerative Disorders. Front Aging Neurosci 7, 248 (2015).

7. Geisler S, et al. PINK1/Parkin-mediated mitophagy is dependent on VDAC1 and p62/SQSTM1. Nat Cell Biol 12, 119-131 (2010).

8. Matsuda N, et al. PINK1 stabilized by mitochondrial depolarization recruits Parkin to damaged mitochondria and activates latent Parkin for mitophagy. J Cell Biol 189, 211 $221(2010)$.

501

10. Tanaka A, et al. Proteasome and p97 mediate mitophagy and degradation of mitofusins induced by Parkin. J Cell Biol 191, 1367-1380 (2010).

11. Chan NC, et al. Broad activation of the ubiquitin-proteasome system by Parkin is critical for mitophagy. Hum Mol Genet, (2011).

12. Lee JY, Nagano Y, Taylor JP, Lim KL, Yao TP. Disease-causing mutations in Parkin impair mitochondrial ubiquitination, aggregation, and HDAC6-dependent mitophagy. $J$ Cell Biol 189, 671-679 (2010). 
13. Yoshii SR, Kishi C, Ishihara N, Mizushima N. Parkin mediates proteasome-dependent protein degradation and rupture of the outer mitochondrial membrane. J Biol Chem 286, 19630-19640 (2011).

14. Wong YC, Holzbaur EL. Optineurin is an autophagy receptor for damaged mitochondria in parkin-mediated mitophagy that is disrupted by an ALS-linked mutation. Proc Natl Acad Sci U S A 111, E4439-4448 (2014).

15. Lazarou $\mathrm{M}$, et al. The ubiquitin kinase PINK1 recruits autophagy receptors to induce mitophagy. Nature 524, 309-314 (2015).

16. Pankiv S, et al. p62/SQSTM1 binds directly to Atg8/LC3 to facilitate degradation of ubiquitinated protein aggregates by autophagy. J Biol Chem 282, 24131-24145 (2007).

17. Narendra D, Kane LA, Hauser DN, Fearnley IM, Youle RJ. p62/SQSTM1 is required for Parkin-induced mitochondrial clustering but not mitophagy; VDAC1 is dispensable for both. Autophagy 6, 1090-1106 (2010).

18. Okatsu K, et al. p62/SQSTM1 cooperates with Parkin for perinuclear clustering of depolarized mitochondria. Genes Cells 15, 887-900 (2010).

19. Itakura E, Kishi-Itakura C, Koyama-Honda I, Mizushima N. Structures containing Atg9A and the ULK1 complex independently target depolarized mitochondria at initial stages of Parkin-mediated mitophagy. J Cell Sci 125, 1488-1499 (2012).

20. Yang JY, Yang WY. Bit-by-bit autophagic removal of parkin-labelled mitochondria. Nat Commun 4, 2428 (2013).

21. Vives-Bauza C, et al. PINK1-dependent recruitment of Parkin to mitochondria in mitophagy. Proc Natl Acad Sci US A 107, 378-383 (2010).

22. Hayashida K, Oyanagi S, Mizutani Y, Yokochi M. An early cytoplasmic change before Lewy body maturation: an ultrastructural study of the substantia nigra from an autopsy case of juvenile parkinsonism. Acta Neuropathol 85, 445-448 (1993).

23. Gai WP, Yuan HX, Li XQ, Power JT, Blumbergs PC, Jensen PH. In situ and in vitro study of colocalization and segregation of alpha-synuclein, ubiquitin, and lipids in Lewy bodies. Exp Neurol 166, 324-333 (2000).

24. Norris KL, et al. Convergence of parkin, PINK1 and alpha-synuclein on stress-induced mitochondrial morphological remodelling. J Biol Chem, (2015). 
25. Choi SY, Huang P, Jenkins GM, Chan DC, Schiller J, Frohman MA. A common lipid links Mfn-mediated mitochondrial fusion and SNARE-regulated exocytosis. Nat Cell Biol 8, 1255-1262 (2006).

26. Huang $\mathrm{H}$, et al. piRNA-associated germline nuage formation and spermatogenesis require MitoPLD profusogenic mitochondrial-surface lipid signaling. Dev Cell 20, 376-387 (2011).

27. Rizzo MA, Shome K, Watkins SC, Romero G. The recruitment of Raf-1 to membranes is mediated by direct interaction with phosphatidic acid and is independent of association with Ras. J Biol Chem 275, 23911-23918 (2000).

28. Giorgione JR, Lin JH, McCammon JA, Newton AC. Increased membrane affinity of the $\mathrm{C} 1$ domain of protein kinase Cdelta compensates for the lack of involvement of its $\mathrm{C} 2$ domain in membrane recruitment. J Biol Chem 281, 1660-1669 (2006).

29. Kassas N, et al. Genetically encoded probes for phosphatidic acid. Methods Cell Biol 108, 445-459 (2012).

30. Baron CL, Malhotra V. Role of diacylglycerol in PKD recruitment to the TGN and protein transport to the plasma membrane. Science 295, 325-328 (2002).

31. Lavieri RR, et al. Design, synthesis, and biological evaluation of halogenated N-(2-(4oxo-1-phenyl-1,3,8-triazaspiro[4.5]decan-8-yl)ethyl)benzamides: discovery of an isoform-selective small molecule phospholipase D2 inhibitor. J Med Chem 53, 67066719 (2010).

32. Han GS, Wu WI, Carman GM. The Saccharomyces cerevisiae Lipin homolog is a Mg2+dependent phosphatidate phosphatase enzyme. J Biol Chem 281, 9210-9218 (2006).

33. Hao R, et al. Proteasomes activate aggresome disassembly and clearance by producing unanchored ubiquitin chains. Mol Cell 51, 819-828 (2013).

34. Shariff A, Luna EJ. Diacylglycerol-stimulated formation of actin nucleation sites at plasma membranes. Science 256, 245-247 (1992).

35. Taunton J, et al. Actin-dependent propulsion of endosomes and lysosomes by recruitment of N-WASP. J Cell Biol 148, 519-530 (2000).

36. Riedl J, et al. Lifeact: a versatile marker to visualize F-actin. Nat Methods 5, 605-607 (2008). 
37. Adachi E, et al. A technique for monitoring multiple signals with a combination of prismbased total internal reflection fluorescence microscopy and epifluorescence microscopy. Pflugers Arch 459, 227-234 (2009).

38. Zhang P, Verity MA, Reue K. Lipin-1 regulates autophagy clearance and intersects with statin drug effects in skeletal muscle. Cell Metab 20, 267-279 (2014).

39. Sahlender DA, et al. Optineurin links myosin VI to the Golgi complex and is involved in Golgi organization and exocytosis. J Cell Biol 169, 285-295 (2005).

40. Morriswood B, et al. T6BP and NDP52 are myosin VI binding partners with potential roles in cytokine signalling and cell adhesion. J Cell Sci 120, 2574-2585 (2007).

41. Zhang C, Li A, Gao S, Zhang X, Xiao H. The TIP30 protein complex, arachidonic acid

42. Takahashi Y, et al. Bif-1 regulates Atg9 trafficking by mediating the fission of Golgi membranes during autophagy. Autophagy 7, 61-73 (2011).

43. Lee JY, et al. HDAC6 controls autophagosome maturation essential for ubiquitinselective quality-control autophagy. Embo J 29, 969-980 (2010).

44. Li S, et al. Transient assembly of F-actin on the outer mitochondrial membrane contributes to mitochondrial fission. J Cell Biol 208, 109-123 (2015).

45. Ji WK, Hatch AL, Merrill RA, Strack S, Higgs HN. Actin filaments target the oligomeric maturation of the dynamin GTPase Drp1 to mitochondrial fission sites. Elife 4, e11553 (2015).

46. Karbowski M, Jeong SY, Youle RJ. Endophilin B1 is required for the maintenance of mitochondrial morphology. J Cell Biol 166, 1027-1039 (2004).

47. Kast DJ, Zajac AL, Holzbaur EL, Ostap EM, Dominguez R. WHAMM Directs the Arp2/3 Complex to the ER for Autophagosome Biogenesis through an Actin Comet Tail Mechanism. Curr Biol 25, 1791-1797 (2015).

48. Coutts AS, La Thangue NB. Actin nucleation by WH2 domains at the autophagosome. Nat Commun 6, 7888 (2015).

49. Bedford L, et al. Depletion of 26S proteasomes in mouse brain neurons causes neurodegeneration and Lewy-like inclusions resembling human pale bodies. $J$ Neurosci 28, 8189-8198 (2008). 
50. Goedert M. Alpha-synuclein and neurodegenerative diseases. Nat Rev Neurosci 2, 492$501(2001)$.

51. Cooper AA, et al. Alpha-synuclein blocks ER-Golgi traffic and Rab1 rescues neuron loss in Parkinson's models. Science 313, 324-328 (2006).

52. Lin X, et al. Leucine-rich repeat kinase 2 regulates the progression of neuropathology induced by Parkinson's-disease-related mutant alpha-synuclein. Neuron 64, 807-827 (2009).

53. MacLeod DA, et al. RAB7L1 interacts with LRRK2 to modify intraneuronal protein sorting and Parkinson's disease risk. Neuron 77, 425-439 (2013).

54. Beilina A, et al. Unbiased screen for interactors of leucine-rich repeat kinase 2 supports a common pathway for sporadic and familial Parkinson disease. Proc Natl Acad Sci US A 111, 2626-2631 (2014).

55. Zavodszky E, et al. Mutation in VPS35 associated with Parkinson's disease impairs WASH complex association and inhibits autophagy. Nat Commun 5, 3828 (2014).

56. Davids $\mathrm{M}$, et al. Disruption of Golgi morphology and altered protein glycosylation in PLA2G6-associated neurodegeneration. J Med Genet 53, 180-189 (2016).

57. Takahashi Y, et al. Bif-1 haploinsufficiency promotes chromosomal instability and accelerates Myc-driven lymphomagenesis via suppression of mitophagy. Blood 121, 1622-1632 (2013).

58. Hung YH, Chen LM, Yang JY, Yang WY. Spatiotemporally controlled induction of autophagy-mediated lysosome turnover. Nat Commun 4, 2111 (2013).

59. Shahnazari S, et al. A diacylglycerol-dependent signaling pathway contributes to regulation of antibacterial autophagy. Cell Host Microbe 8, 137-146 (2010).

60. Thurston TL, Ryzhakov G, Bloor S, von Muhlinen N, Randow F. The TBK1 adaptor and autophagy receptor NDP52 restricts the proliferation of ubiquitin-coated bacteria. Nat Immunol 10, 1215-1221 (2009).

61. Tan BK, Bogdanov M, Zhao J, Dowhan W, Raetz CR, Guan Z. Discovery of a cardiolipin synthase utilizing phosphatidylethanolamine and phosphatidylglycerol as substrates. Proc Natl Acad Sci U S A 109, 16504-16509 (2012). 
672 We thank Drs. M. Frohman and I. Kojima for PA and DAG reporters, W. G. Zhang for PLD1/2-

673 YFP, E.F Holzbaur for OPTN-Cherry, and V. Bennett for TGN38-GFP expression plasmids. Dr.

674 M Frohman for PLD6 KO MEFs. We thank Dr. T. Slotkin for the advice on the statistical

675 analysis and YS Gao on the image analysis. This work was supported by 2R01-NS054022 (NIH)

676 to T.-P.Y.

677 Author contributions

678 K.L.S and T.P.Y. conceived the project initially. C.C.L, J.Y., M.D.K. and K.L.N designed and

679 performed the majority of the experiments. Z.Q performed the lipidomic analysis. C.W.H.

680 performed the live-cell imaging experiments. C.H.L. assisted with reagent preparation and 681 experiments. T.P.Y. J.T.C and W.Y.Y supervised the work. N.V. and K.L.L. collaborated in the 682 discussion and provided critical reagents. C.C.L., J.Y., M.D.K and T.P.Y. wrote the manuscript.

683 Competing interests: The authors declare no competing financial interests. 


\section{Abbreviations List}

687 Outer-mitochondrial membrane (OMM), inter-membrane space (IMS), inner-mitochondrial

688 membrane (IMM), phosphatidic acid (PA), diacylglycerol (DAG), optineurin (OPTN),

689 endophilin b1 (EndoB1), carbonyl chloro-m-phenylhydrazine (CCCP), phospholipase D 6

690 (mitoPLD), PTEN-inducible kinase 1 (PINK1).

691

692

693

694

695

696

697

698

699

700

701

702

703

704

705

706

707

708

709

710

711

712

713

714

715

716

717

718

719

720

721

722 


\section{$723 \quad$ Figure Legends}

724 Fig. 1 Parkin stimulates PA and DAG accumulation on depolarized mitochondria. HeLa

725 cells were transfected with a WT or mutant Parkin plasmid (FLAG), shown in blue, along with

726 reporters for either PA or DAG (see Materials and Methods), shown in green. DMSO or $10 \mu \mathrm{M}$

727 CCCP was added for the indicated periods of time. Mitochondria were visualized via anti-

728 Tom20 staining (red). a PA accumulated on Parkin-positive mitochondria after 1 hour of 10uM

729 CCCP treatment, with a more dramatic increase by $3 \mathrm{hr}$ and $18 \mathrm{hr}$. b DAG (diacylglycerol)

730 accumulated on Parkin-positive mitochondria after $5.5 \mathrm{hr}$ of $10 \mathrm{uM}$ CCCP treatment. c

731 Quantification of imaging experiments shown in A and B. d E3 ligase-deficient Parkin mutants

732 (T240R and T415N) have no effect on PA accumulation (green) on depolarized, Parkin-positive

733 mitochondria. e DAG accumulation depends upon intact Parkin E3 ligase activity. Scale bar =

$73425 \mu \mathrm{m}$ and zoom is $3 \mathrm{x}$. f Quantification of imaging experiments shown in D and E. Bars

735 represent mean with SEM from three independent experiments; two-way ANOVA analysis was

736 performed for statistical analysis $(* * * \mathrm{P}<0.0001)$.

737 Fig. 2 PLD2 and lipin1 work in tandem to stimulate mitochondrial PA and DAG

738 production. a HeLa cells were transfected with expression plasmids for Parkin (mCherry-

739 Parkin, Red) and YFP-PLD2 (Green) and treated with DMSO or $10 \mu \mathrm{M}$ CCCP for the indicated

740 time. Mitochondria were visualized via anti-Tom20 staining (blue). Note that YFP-PLD2

741 accumulated on Parkin-positive mitochondria after CCCP treatment. Scale bar $=25 \mu \mathrm{m}$. b HEK-

742 293T cells were transfected with Parkin-FLAG and DAGR-YFP followed by CCCP treatment

743 for indicated times. Mitochondrial and cytosolic fractions were purified and analyzed by Western 
744 blots by indicated antibodies. Citrate synthetase and $\alpha$-tubulin were used as a mitochondrial and

745 cytosolic marker, respectively. Note that PLD2 and Parkin levels were elevated in the

746 mitochondrial fraction in response to CCCP treatment. c Hela cells were co-transfected with

747 mcherry-Parkin and the PA reporter, followed by CCCP treatment alone or with a PLD2

748 inhibitor VU $0364739(3 \mu \mathrm{M})$ for $5.5 \mathrm{hrs}$. Line scan analysis (Image J software) corresponding to

749 the line drawn in the images indicate colocalization between the PA reporter (green) and

750 mitochondria (red). Note that VU 0364739 suppressed mitochondrial PA accumulation. Scale

751 bar $=10 \mu \mathrm{m}$. d Quantification of the numbers of cells with the PA reporter positive mitochondria

752 shown in c. Asterisks indicate statistical significance (***P $<0.001$, Student's t-test). e Hela cells

753 were transfected with a lipin1 siRNA, followed by the DAG reporter and mCherry-Parkin,

754 treated with CCCP $(10 \mu \mathrm{M})$ for $9 \mathrm{hrs}$ and then subject to image analysis. Scale bar $=25$ um and

755 zoom is $4 x$. f The numbers of cells with DAG-reporter positive mitochondria shown in $\mathbf{e}$ and in

756 Lipin-1 knockdown cells transfected with siRNA-resistant wildtype (WT) and catalytically dead

757 (CD; D712E;D714E) mutant cDNA. Note that Lipin1 KD reduced mitochondrial DAG

758 accumulation, which can be significantly restored by the re-expression of WT, but not CD

759 mutant, Lipin-1. The graph shows the means with SEM (error bars) from three independent

760 experiments. Asterisks indicate statistical significance by one-way ANOVA (**P< 0.01 ,

$761 * * * \mathrm{P}<0.001)$. Source data are provided as a Source Data file.

762 Fig. 3 Lipin1 regulates mitophagy and F-actin-dependent mitochondrial dispersion. a,b

763 Hela cells were transfected with a Lipin1 or control (cKD) siRNA followed by a GFP-Parkin

764 expression plasmid. Transfected cells were treated with CCCP at $10 \mu \mathrm{M}$ for $18 \mathrm{hrs}$, and subject 
765 to immuno-staining. b Quantification of Parkin-positive cells in a that have lost a majority of 766 mitochondria (marked by TOM20, arrows) from two independent experiments. Asterisks

767 indicate statistical significance $(* \mathrm{P}<0.05$, Student's t-test). Note that mitochondrial clearance is 768 reduced in Lipin-1 knockdown cells (arrowheads in a). c Control or lipin1 knockdown Hela cells 769 stably expressing parkin-mCherry were treated with DMSO or CCCP (10 $\mu \mathrm{M}$ for 18 hrs $)$ and 770 Bafilomycin A1 (1 $1 \mu \mathrm{M}$, lysosomal inhibitor) treatment, followed by immunoblotting with 771 indicated antibodies. Note that lipin1 silencing rescued mitochondrial protein degradation. The 772 band intensity of mitochondrial proteins relative to control untreated conditions were determined 773 by Image $\mathrm{J}$. d HeLa cells were treated and processed as described in a but with 9 hr CCCP 774 treatment. Small and dispersed mitochondria were prominent in control knockdown cells while 775 this dispersion was suppressed in Lipin-1 knockdown cells. e Quantification of the number of 776 dispersed mitochondria, as shown in $\mathbf{d}$. f-h Control or Lipin-1 knockdown Hela cells were co777 transfected with GFP-Parkin, a mitochondrial marker (CFP-Mito), and F-actin marker (RFP778 LifeAct) followed by CCCP treatment alone or with Cytochalasin B at $10 \mu \mathrm{M}$, as indicated.

779 Live cell images were then acquired and analyzed. Note the marked co-localization of RFP780 LifeAct and Parkin-positive mitochondria in control but not Cytochalasin B treated $\mathbf{f}$ or Lipin-1

781 KD cells $\mathbf{~ h . ~} \mathbf{g}$ Quantification of the effect of Cytochalasin B on mitochondrial dispersion from $\mathbf{f}$.

782 Scale bar $=25 \mu \mathrm{M}$ and zoom is $3 \mathrm{x} \mathbf{d}$ or $12 \mathrm{x} \mathbf{f}$ or $7 \mathrm{x} \mathbf{h}$. Source data are provided as a Source Data 783 file.

784 Fig. 4 Lipin-1-mediated mitochondrial DAG production is required for mitophagosome

785 production. Hela cells were transfected with control or a Lipin1 siRNA followed by the 
expression plasmids of GFP-Parkin and a DAG reporter (RFP-DAGR). a,b Transfected cells were treated with DMSO a or CCCP $\mathbf{b}$ at $10 \mu \mathrm{M}$ for $9 \mathrm{hr}$ as indicated. Autophagosome formation was assessed by a LC3 antibody. LC3 is pseudo colored in white in single channel and blue in the overlay. c Quantification of autophagosome (LC3) from experiment in a, where at least 50

790 cells in three independent experiments were analyzed. d Lipin1 knockdown cells were further

791 incubated with 1,2-Dipalmitoyl-sn-glycerol (DPG) at $100 \mu \mathrm{M}$ in addition to CCCP and assessed

792 for autophagosome formation. Note that CCCP induced DAG-positive autophagosomes that

793 sequester dispersed mitochondria in control knockdown cells and this induction is suppressed in

794 Lipin-1 knockdown cells (9 hr post CCCP). 1,2-Dipalmitoyl-sn-glycerol treatment induced

795 DAG-positive autophagosomes in Lipin-1 knockdown cells. Scale bar $=25 \mu \mathrm{M}$ and zoom is $5 \mathrm{x}$.

796 e,f The number e and average size (f, arbitrary unit) of LC3-vesicles from indicated samples in d

797 were quantified by Image J software. Note that both the number and size of LC3-vesicles in

798 Lipin-1 knockdown cells were much smaller than those in WT cells and these defects were

799 corrected by DPG treatment. Asterisks indicate statistical significance by one-way ANOVA ( ${ }^{*} \mathrm{P}<$

$800 \quad 0.05, * * \mathrm{P}<0.01)$ from four independent experiments. Source data are provided as a Source Data

801 file.

802 Fig. 5 Requirement of OPTN, NDP52 for DAG production and exogenous DAG restores

803 mitophagy. a Hela cells were transfected with control or optineurin and NDP52 siRNAs

804 followed by expression plasmids of FLAG-Parkin a with the YFP-DAGR. These cells were

805 treated with DMSO or $10 \mu \mathrm{M}$ CCCP for $5.5 \mathrm{hrs}$, as indicated. Note that OPTN/NDP52 double

806 knockdown prevented mitochondrial YFP-DAGR accumulation. b Hela OPTN/NDP52 
807 knockdown cells were transfected with a siRNA-resistant wildtype or ubiquitin-binding deficient

808 E478G OPTN mutant, as indicated. The percentage of cells with mitochondrial YFP-DAGR

809 following CCCP treatment was quantified. Asterisks indicate statistical significance by one-way

810 ANOVA $(* * \mathrm{P}<0.01, * * * \mathrm{P}<0.001)$ from three independent experiments. c Hela OPTN/NDP52

811 knockdown cells were generated as a and incubated with 1,2-Dipalmitoyl-sn-glycerol followed

812 by CCCP treatment for 18 hrs. Note that mitophagy was markedly restored by 1,2-Dipalmitoyl-

813 sn-glycerol in OPTN/NDP52 knockdown cells. d Quantification of percentage of cells with

814 cleared mitochondrial in c. Asterisks indicate statistical significance $(* \mathrm{P}<0.05, * * \mathrm{P}<0.01$,

815 Student's t-test) from two independent experiments. Scale bar $=25 \mu \mathrm{M}$ and zoom is $3 \mathrm{x}$. Source

816 data are provided as a Source Data file.

817 Fig. 6 OPTN and NDP52 deliver EndoB1-positive golgi vesicles to ubiquitinated

818 mitochondria for mitochondrial DAG production. a,b Hela cells were co-transfected with

819 expression plasmids for GFP-TGN38, mCherry-OPTN and FLAG-Parkin a or mCherry-OPTN

820 and GFP-Parkin b and treated with CCCP for 5.5 hrs. Mitochondria were visualized by

821 immunostaining with a Tom20 antibody and EndoB1 localization was assessed by an EndoB1

822 antibody. Note that GFP-TGN38 (a bottom panel) and EndoB1 (b bottom panel), pseudo colored

823 in white in single channel and blue in the overlay images, translocates to mitochondria. c

824 Mitochondrial and cytosolic fractions obtained from control and CCCP treated cells, as was

825 described and analyzed in Fig. 2b, were immunoblotted for EndoB1 and OPTN, as indicated. d

826 Hela OPTN/NDP52 knockdown cells were transfected with YFP-DAGR and mCherry-Parkin

827 and treated with $\mathrm{CCCP}$ as indicated. EndoB1 localization was assessed by an EndoB1 antibody 
828 and was presented in pseudo color white as $\mathbf{b}$. Note that translocation of EndoB1 to mitochondria

829 was impaired in OPTN/NDP52 knockdown cells (bottom panel). e Cytosolic and mitochondrial

830 fractions purified from control and OPTN/NDP52 knockdown cells treated with CCCP were

831 immunoblotted for antibodies, as indicated. Note that mitochondrial EndoB1 levels were reduced

832 in OPTN/NDP52 knockdown cells. f,g Hela cells were transfected with an EndoB1 siRNA,

833 followed by transfection of expression plasmids for mCherry-Parkin and YFP-DAGR, and

$834 \mathrm{CCCP}$ treatment at $10 \mu \mathrm{M}$ for $9 \mathrm{hrs}$. Cells with mitochondrial YFP-DAGR were quantified from

$835 \mathrm{f}(* \mathrm{P}<0.05$, Student's t-test $)$. Scale bar $=25 \mu \mathrm{M}$ and zoom is $5 \mathrm{x}$. Note that knockdown of

836 EndoB1 suppressed mitochondrial DAG production. 


\section{Lin_Figure 1}

a
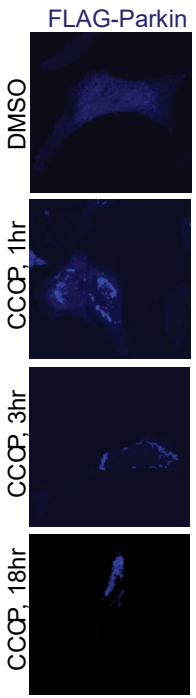

b
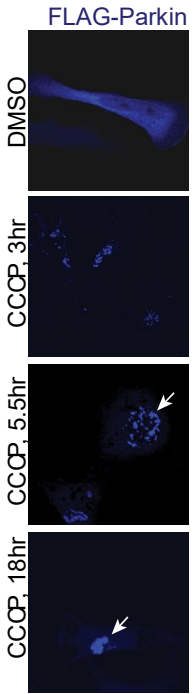
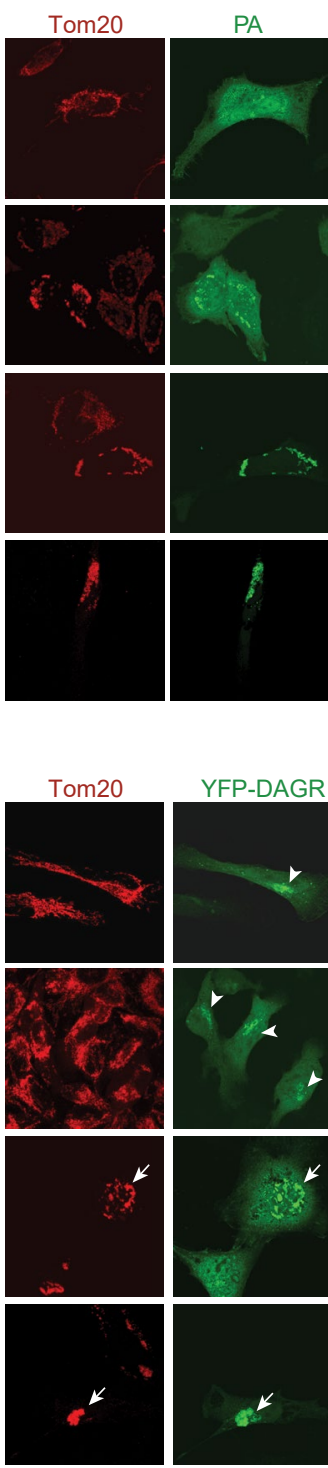
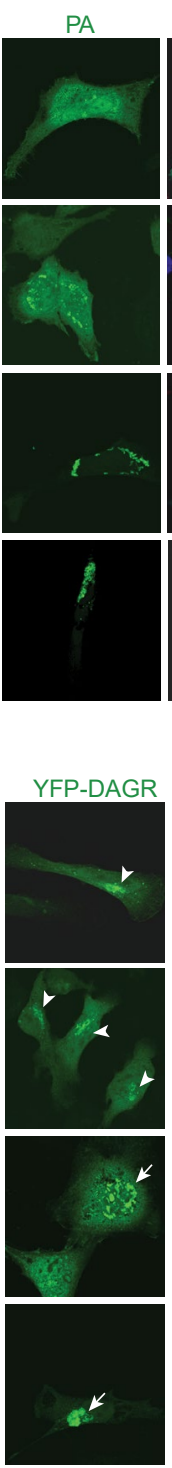

Overlay
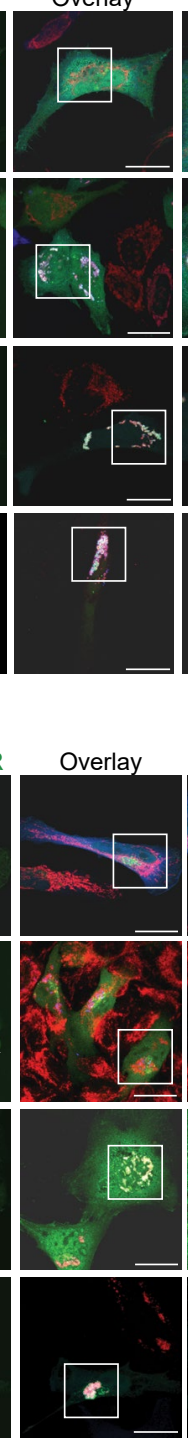

Zoom

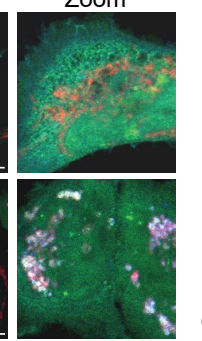

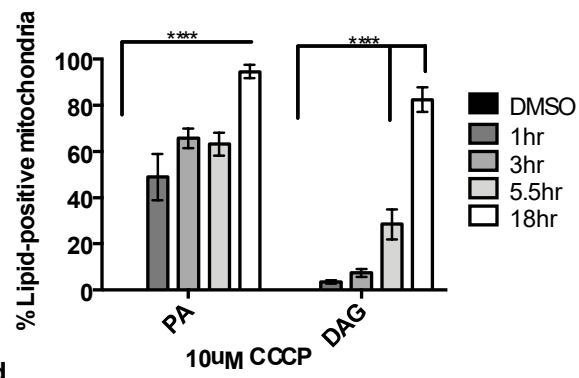

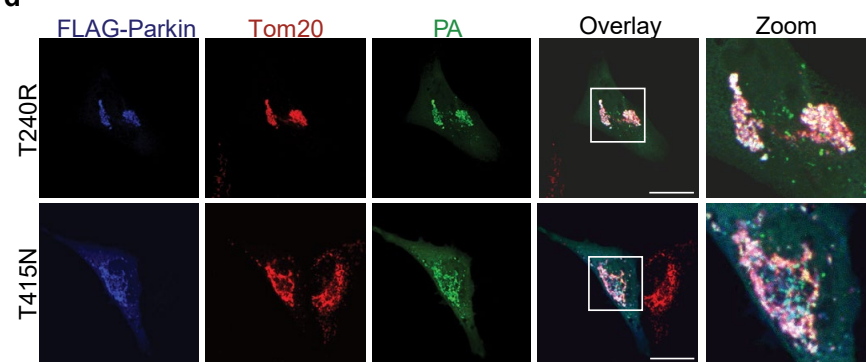

e

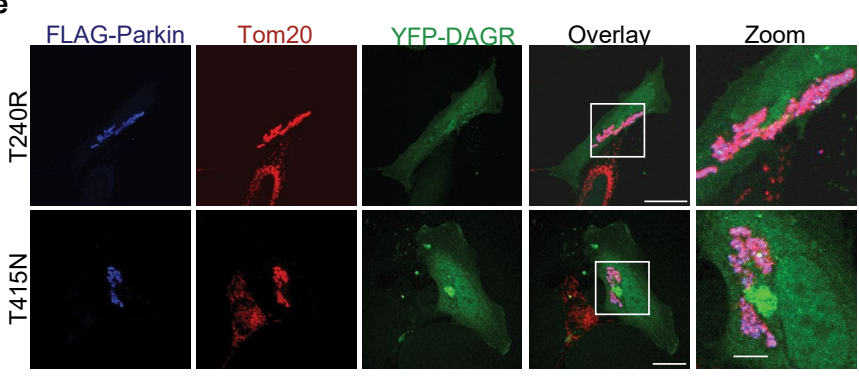

f

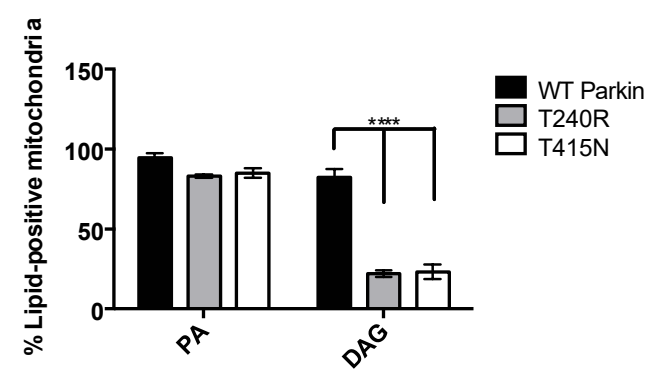

Fig. 1 Parkin stimulates PA and DAG accumulation on depolarized mitochondria. HeLa cells were transfected with a WT or mutant Parkin plasmid (FLAG), shown in blue, along with reporters for either PA or DAG (see Materials and Methods), shown in green. DMSO or $10 \mu \mathrm{M}$ CCCP was added for the indicated periods of time. Mitochondria were visualized via anti-Tom20 staining (red). a PA

accumulated on Parkin-positive mitochondria after 1 hour of 10uM CCCP treatment, with a more dramatic increase by $3 \mathrm{hr}$ and $18 \mathrm{hr}$. b DAG (diacylglycerol) accumulated on Parkin-positive mitochondria after $5.5 \mathrm{hr}$ of $10 \mathrm{uM} \mathrm{CCCP}$ treatment. c Quantification of imaging experiments shown in A and B. d E3 ligase-deficient Parkin mutants (T240R and T415N) have no effect on PA accumulation (green) on depolarized, Parkin-positive mitochondria. e DAG accumulation depends upon intact Parkin E3 ligase activity. Scale bar $=25 \mu \mathrm{m}$ and zoom is 3x. $\mathbf{f}$ Quantification of imaging experiments shown in D and $E$. Bars represent mean with SEM from three independent experiments; two-way ANOVA analysis was performed for statistical analysis $\left.{ }^{* * *} \mathrm{P}<0.0001\right)$. 


\section{Lin_Figure 2}

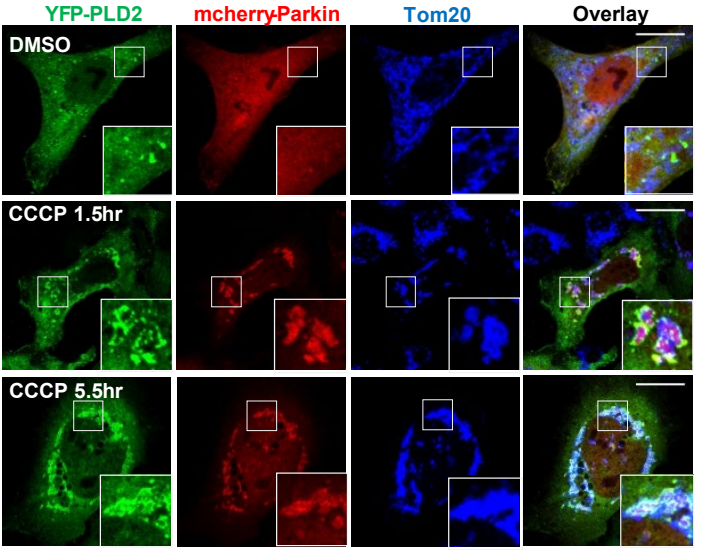

b

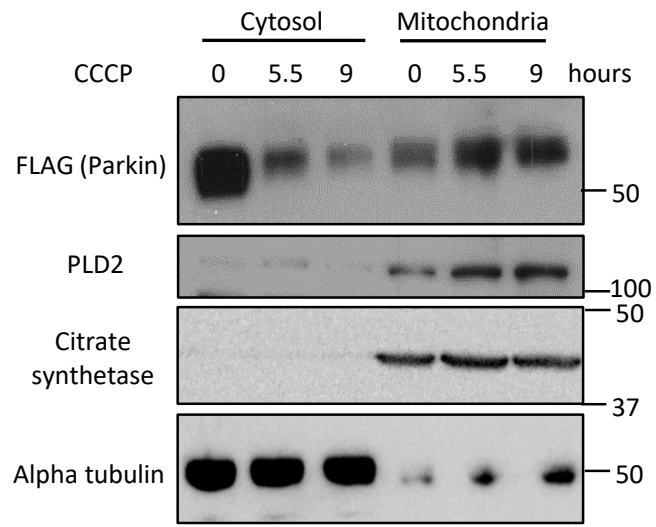

d
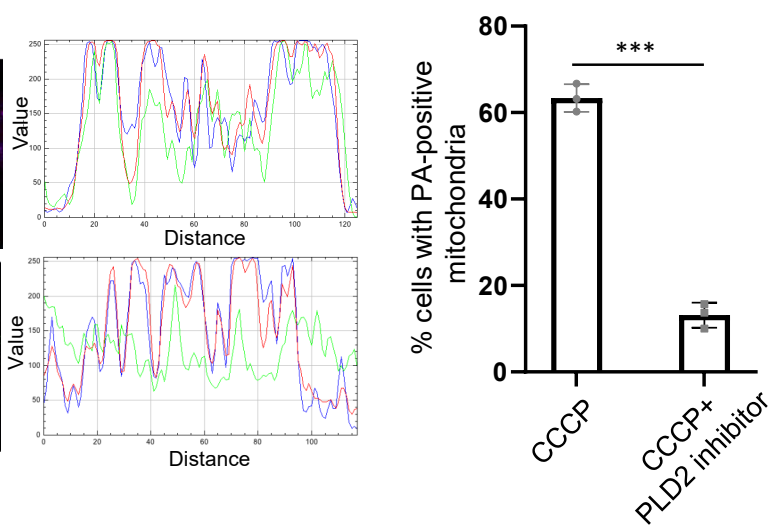

C

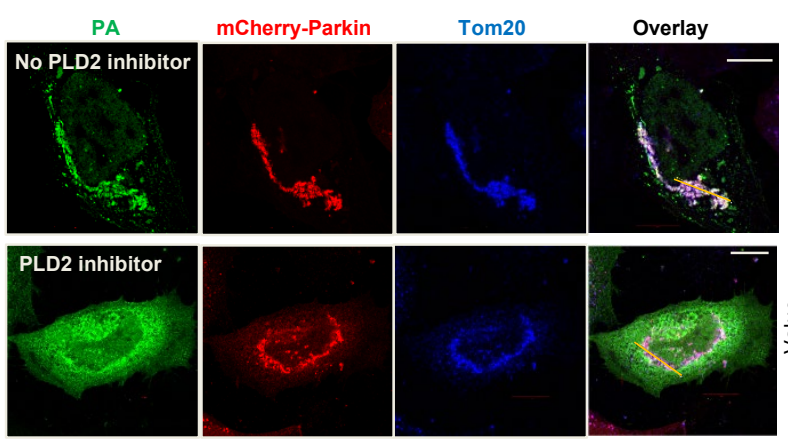

e
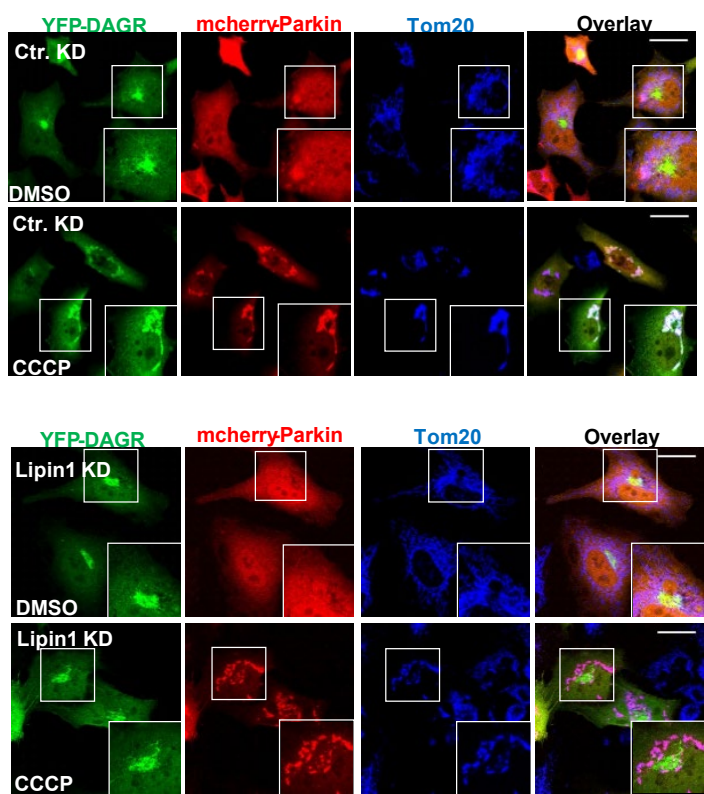

f

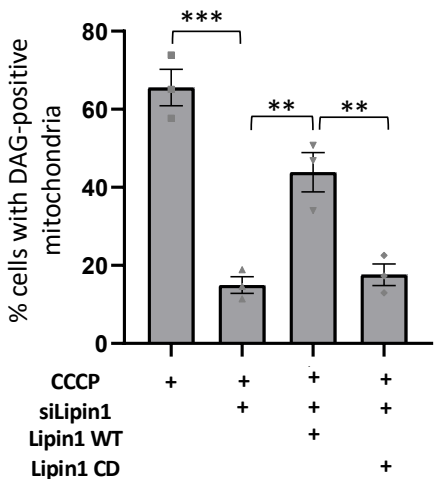

Fig. 2 PLD2 and lipin1 work in tandem to stimulate mitochondrial PA and DAG production. a HeLa cells were transfected with expression plasmids for Parkin (mCherry-Parkin, Red) and YFP-PLD2 (Green) and treated with DMSO or $10 \mu \mathrm{M}$ CCCP for the indicated time. Mitochondria were visualized via anti-Tom20 staining (blue). Note that YFP-PLD2 accumulated on Parkin-positive mitochondria after CCCP treatment. Scale bar $=25 \mu \mathrm{m}$. b HEK-293T cells were transfected with Parkin-FLAG and DAGR-YFP followed by CCCP treatment for indicated times. Mitochondrial and cytosolic fractions were purified and analyzed by Western blots by indicated antibodies. Citrate synthetase and $\alpha$-tubulin were used as a mitochondrial and cytosolic marker, respectively. Note that PLD2 and Parkin levels were elevated in the mitochondrial fraction in response to CCCP treatment. c Hela cells were co-transfected with mcherry-Parkin and the PA reporter, followed by CCCP treatment alone or with a PLD2 inhibitor VU $0364739(3 \mu \mathrm{M})$ for 5.5 hrs. Line scan analysis (Image $\mathrm{J}$ software) corresponding to the line drawn in the images indicate colocalization between the PA reporter (green) and mitochondria (red). Note that VU 0364739 suppressed mitochondrial PA accumulation. Scale bar $=10 \mu \mathrm{m}$. d Quantification of the numbers of cells with the PA reporter positive mitochondria shown in c. Asterisks indicate statistical significance $\left({ }^{\star \star *} P<0.001\right.$, Student's t-test). e Hela cells were transfected with a lipin1 siRNA, followed by the DAG reporter and mCherry-Parkin, treated with CCCP (10 $\mu \mathrm{M})$ for 9 hrs and then subject to image analysis. Scale bar $=25 \mathrm{um}$ and zoom is $4 \mathrm{x}$. $\mathbf{f}$ The numbers of cells with DAG-reporter positive mitochondria shown in e and in Lipin-1 knockdown cells transfected with siRNA-resistant wildtype (WT) and catalytically dead (CD; D712E;D714E) mutant cDNA. Note that Lipin1 KD reduced mitochondrial DAG accumulation, which can be significantly restored by the re-expression of WT, but not CD mutant, Lipin-1. The graph shows the means with SEM (error bars) from three independent experiments. Asterisks indicate statistical significance by one-way ANOVA $\left({ }^{* *} P<0.01,{ }^{* * *} \mathrm{P}<0.001\right)$. Source data are provided as a Source Data file. 


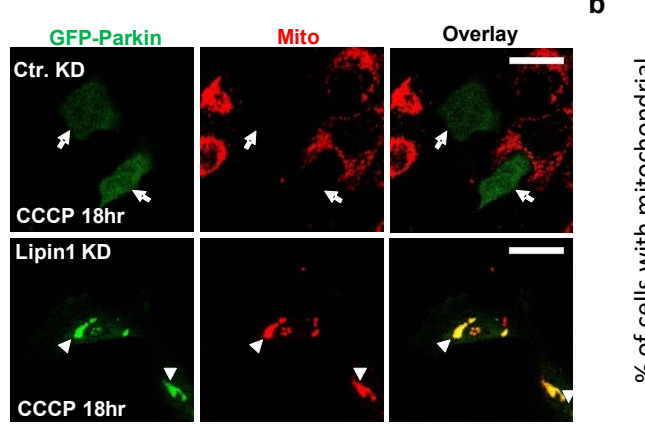

d
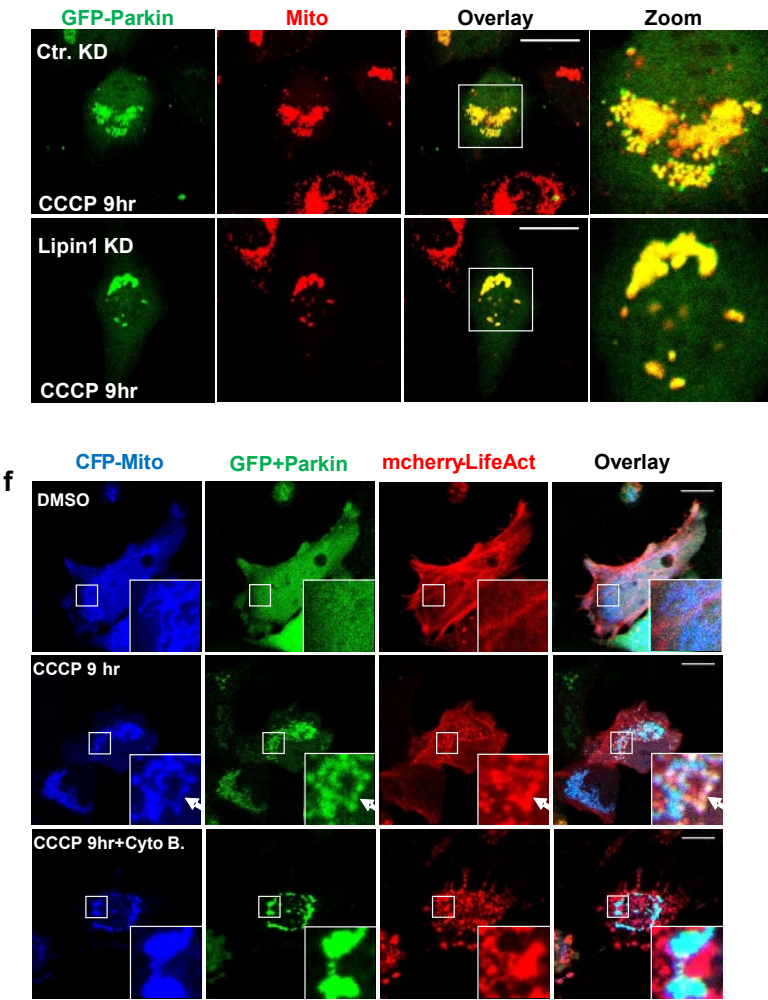

h

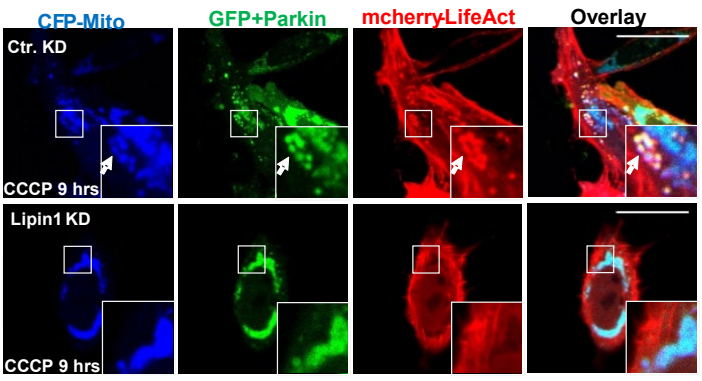

c

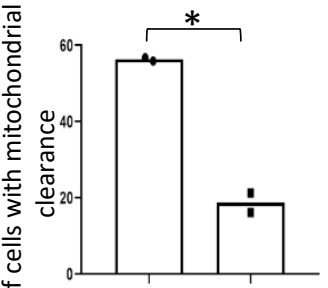

Ctr. KD Lipin1 KD
DMSO

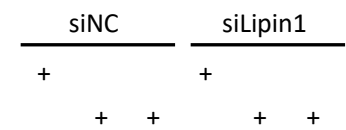

Bafilomycin A1

Lipin1

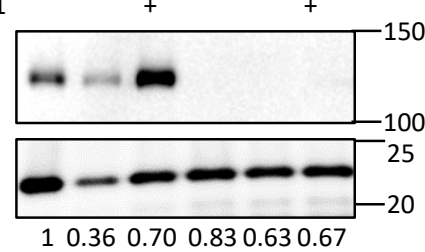

Tim23

Citrate synthetase

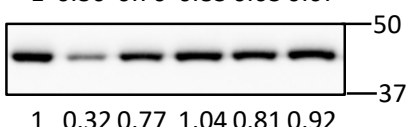

Hsp60

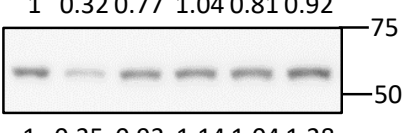

GAPDH

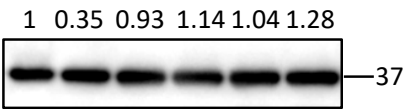

e

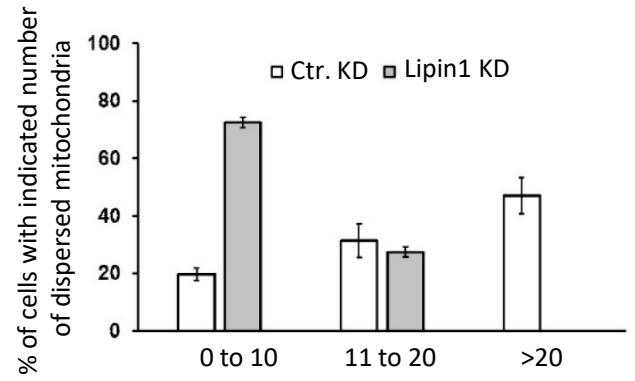

g

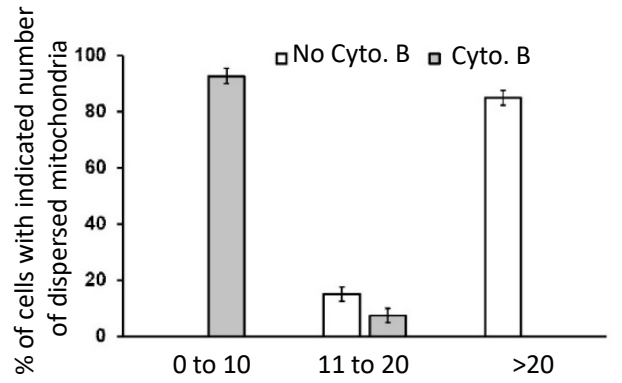

Fig. 3 Lipin1 regulates mitophagy and F-actin-dependent mitochondrial dispersion. a,b Hela cells were transfected with a Lipin1 or control (CKD) siRNA followed by a GFP-Parkin expression plasmid. Transfected cells were treated with CCCP at $10 \mu \mathrm{M}$ for 18 hrs, and subject to immuno-staining. b Quantification of Parkin-positive cells in a that have lost a majority of mitochondria (marked by TOM20, arrows) from two independent experiments. Asterisks indicate statistical significance $\left({ }^{*} \mathrm{P}<0.05\right.$, Student's t-test). Note that mitochondrial clearance is reduced in Lipin-1 knockdown cells (arrowheads in a). c Control or lipin1 knockdown Hela cells stably expressing parkin-

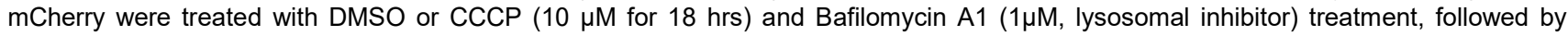
immunoblotting with indicated antibodies. Note that lipin1 silencing rescued mitochondrial protein degradation. The band intensity of mitochondrial proteins relative to control untreated conditions were determined by Image $\mathbf{J}$. $\mathbf{d}$ HeLa cells were treated and processed as described in a but with $9 \mathrm{hr}$ CCCP treatment. Small and dispersed mitochondria were prominent in control knockdown cells while this dispersion was suppressed in Lipin-1 knockdown cells. e Quantification of the number of dispersed mitochondria, as shown in d. f-h Control or Lipin-1 knockdown Hela cells were co-transfected with GFP-Parkin, a mitochondrial marker (CFP-Mito), and F-actin marker (RFP-LifeAct) followed by CCCP treatment alone or with Cytochalasin B at $10 \mu \mathrm{M}$, as indicated. Live cell images were then acquired and analyzed. Note the marked co-localization of RFP-LifeAct and Parkin-positive mitochondria in control but not Cytochalasin B treated $\mathbf{f}$ or Lipin-1 KD cells $\mathbf{h}$. $\mathbf{g}$ Quantification of the effect of Cytochalasin B on mitochondrial dispersion from $\mathbf{f}$. Scale bar $=25 \mu \mathrm{M}$ and zoom is $3 \mathrm{x}$ $\mathbf{d}$ or $12 \mathrm{x} \mathbf{f}$ or $7 \mathbf{x} \mathbf{h}$. Source data are provided as a Source Data file. 


\section{Lin_Figure 4}

a

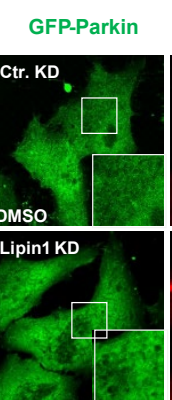

b
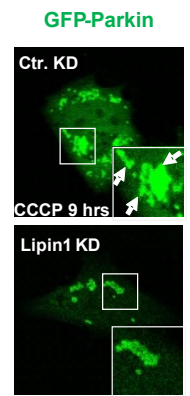

d
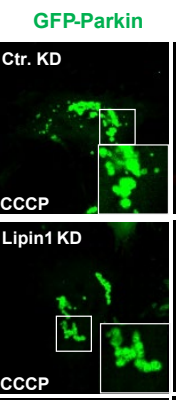

Lipin1 KD+DPG

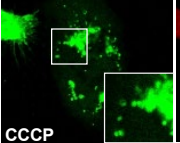

RFP-DAGR

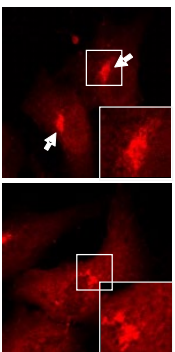

RFP-DAGR

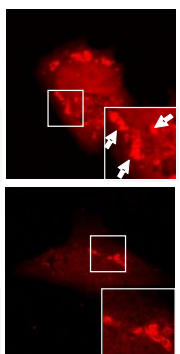

RFP-DAGR
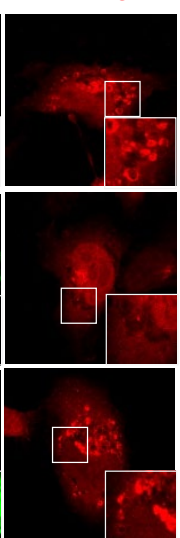

LC3

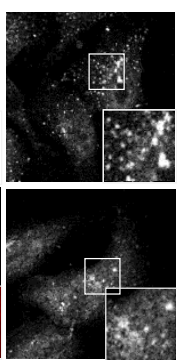

LC3
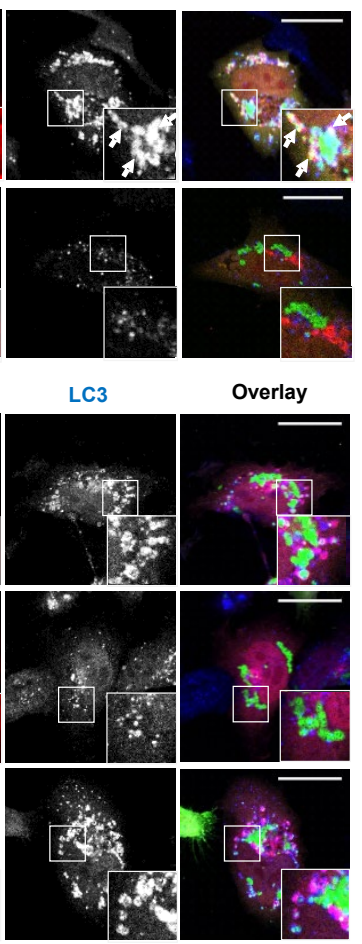

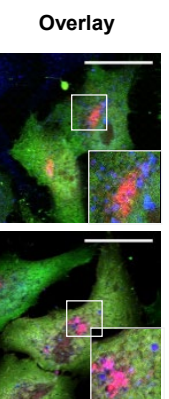

Overlay

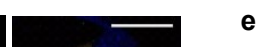

C
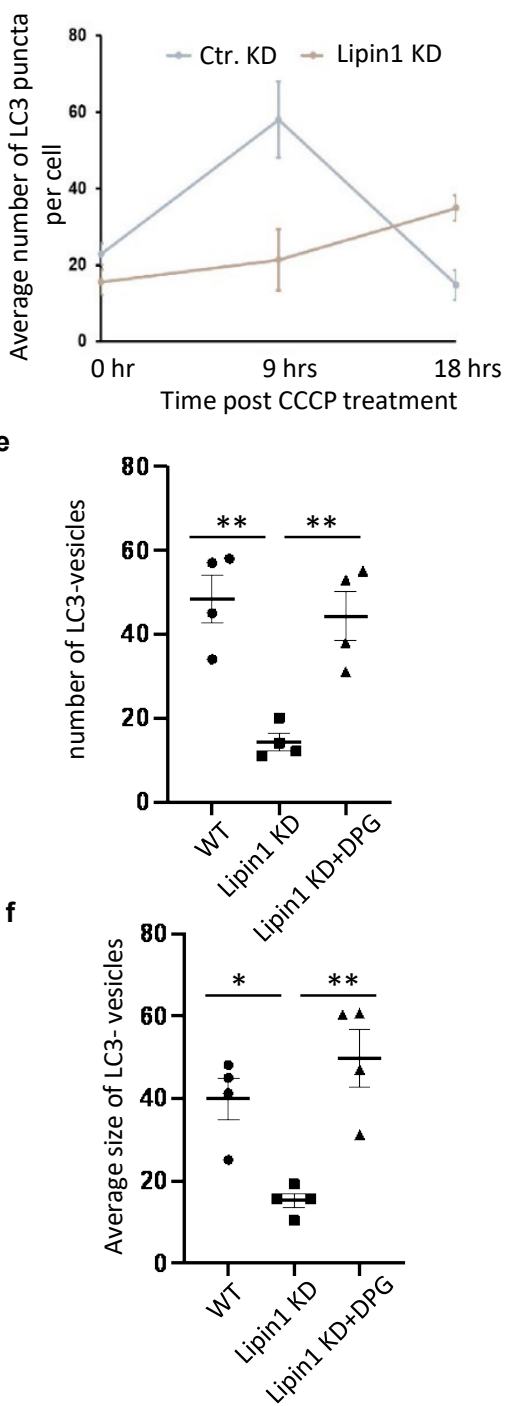

Fig. 4 Lipin-1-mediated mitochondrial DAG production is required for mitophagosome production. Hela cells were transfected with control or a Lipin1 siRNA followed by the expression plasmids of GFP-Parkin and a DAG reporter (RFP-DAGR). a,b Transfected cells were treated with DMSO a or CCCP $\mathbf{b}$ at $10 \mu \mathrm{M}$ for $9 \mathrm{hr}$ as indicated. Autophagosome formation was assessed by a LC3 antibody. LC3 is pseudo colored in white in single channel and blue in the overlay. c Quantification of autophagosome (LC3) from experiment in a, where at least 50 cells in three independent experiments were analyzed. d Lipin1 knockdown cells were further incubated with 1,2-Dipalmitoylsn-glycerol (DPG) at $100 \mu \mathrm{M}$ in addition to CCCP and assessed for autophagosome formation. Note that CCCP induced DAG-positive autophagosomes that sequester dispersed mitochondria in control knockdown cells and this induction is suppressed in Lipin-1 knockdown cells (9 hr post CCCP). 1,2-Dipalmitoyl-sn-glycerol treatment induced DAG-positive autophagosomes in Lipin-1 knockdown cells. Scale bar $=25 \mu \mathrm{M}$ and zoom is $5 x$. e,f The number $\mathbf{e}$ and average size (f, arbitrary unit) of LC3-vesicles from indicated samples in $\mathbf{d}$ were quantified by Image $\mathrm{J}$ software. Note that both the number and size of LC3-vesicles in Lipin-1 knockdown cells were much smaller than those in WT cells and these defects were corrected by DPG treatment. Asterisks indicate statistical significance by one-way ANOVA $\left({ }^{\star} \mathrm{P}<0.05,{ }^{\star \star} \mathrm{P}<0.01\right)$ from four independent experiments. Source data are provided as a Source Data file. 


\section{Lin_Figure 5}

a
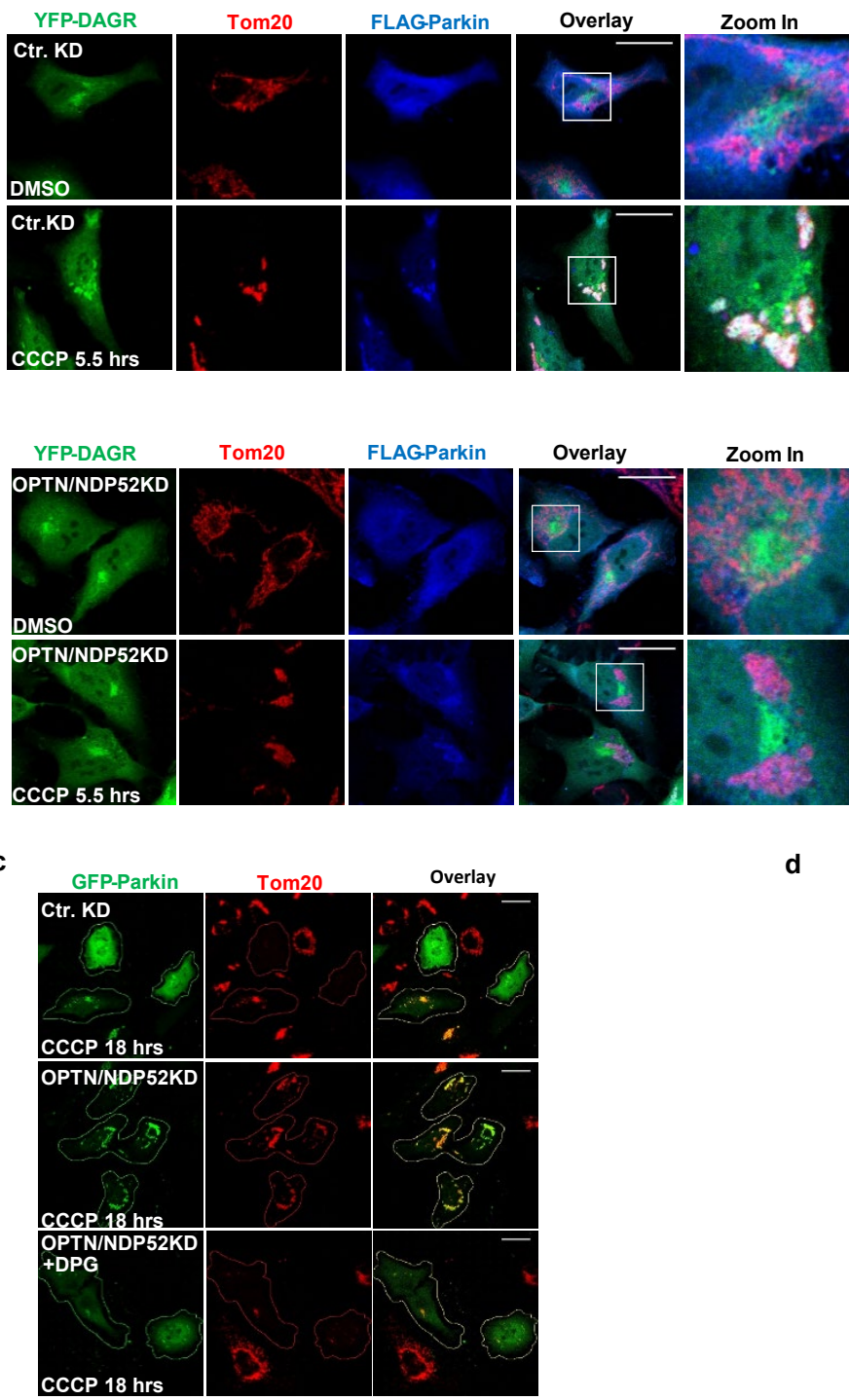

b

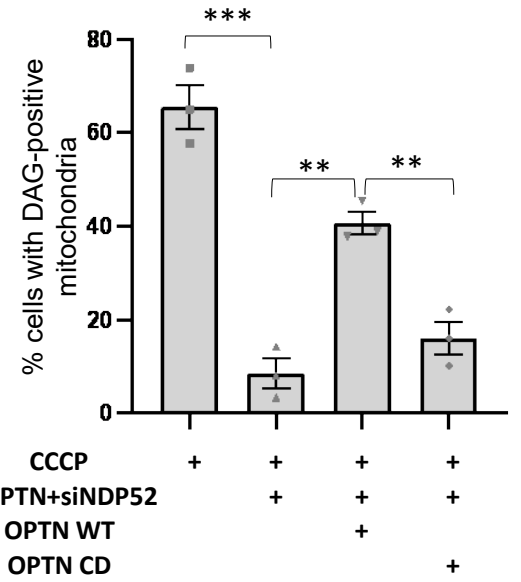

d

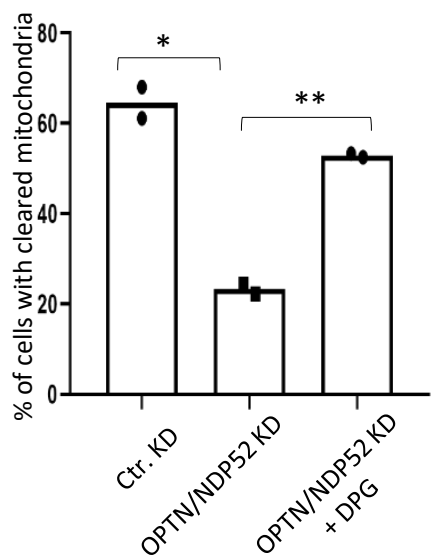

Fig. 5 Requirement of OPTN, NDP52 for DAG production and exogenous DAG restores mitophagy. a Hela cells were transfected with control or optineurin and NDP52 siRNAs followed by expression plasmids of FLAG-Parkin a with the YFP-DAGR. These cells were treated with DMSO or $10 \mu \mathrm{M}$ CCCP for $5.5 \mathrm{hrs}$, as indicated. Note that OPTN/NDP52 double knockdown prevented mitochondrial YFPDAGR accumulation. b Hela OPTN/NDP52 knockdown cells were transfected with a siRNA-resistant wildtype or ubiquitin-binding deficient E478G OPTN mutant, as indicated. The percentage of cells with mitochondrial YFP-DAGR following CCCP treatment was quantified. Asterisks indicate statistical significance by one-way ANOVA $\left({ }^{* *} P<0.01,{ }^{* *} P<0.001\right)$ from three independent experiments. $\mathrm{c}$ Hela OPTN/NDP52 knockdown cells were generated as a and incubated with 1,2-Dipalmitoyl-sn-glycerol followed by CCCP treatment for $18 \mathrm{hrs}$. Note that mitophagy was markedly restored by 1,2-Dipalmitoyl-sn-glycerol in OPTN/NDP52 knockdown cells. d Quantification of percentage of cells with cleared mitochondrial in c. Asterisks indicate statistical significance $\left({ }^{*} P<0.05\right.$, ${ }^{* *} P<0.01$, Student's t-test) from two independent experiments. Scale bar $=25 \mu \mathrm{M}$ and zoom is $3 \mathrm{x}$. Source data are provided as a Source Data file. 


\section{Lin_Figure 6}

a

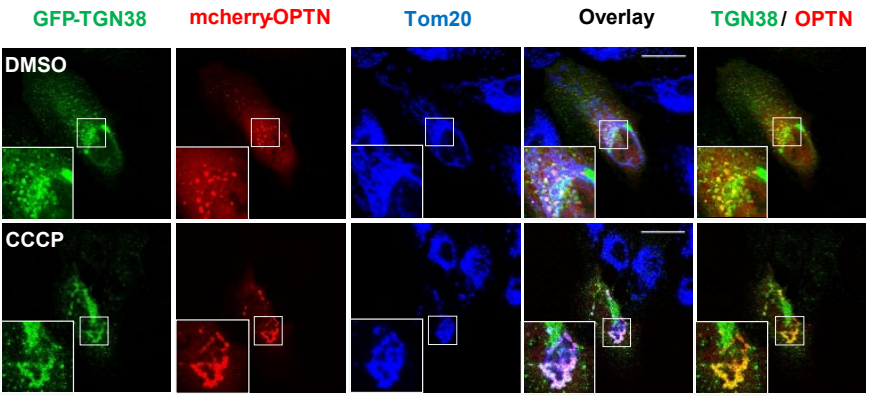

c
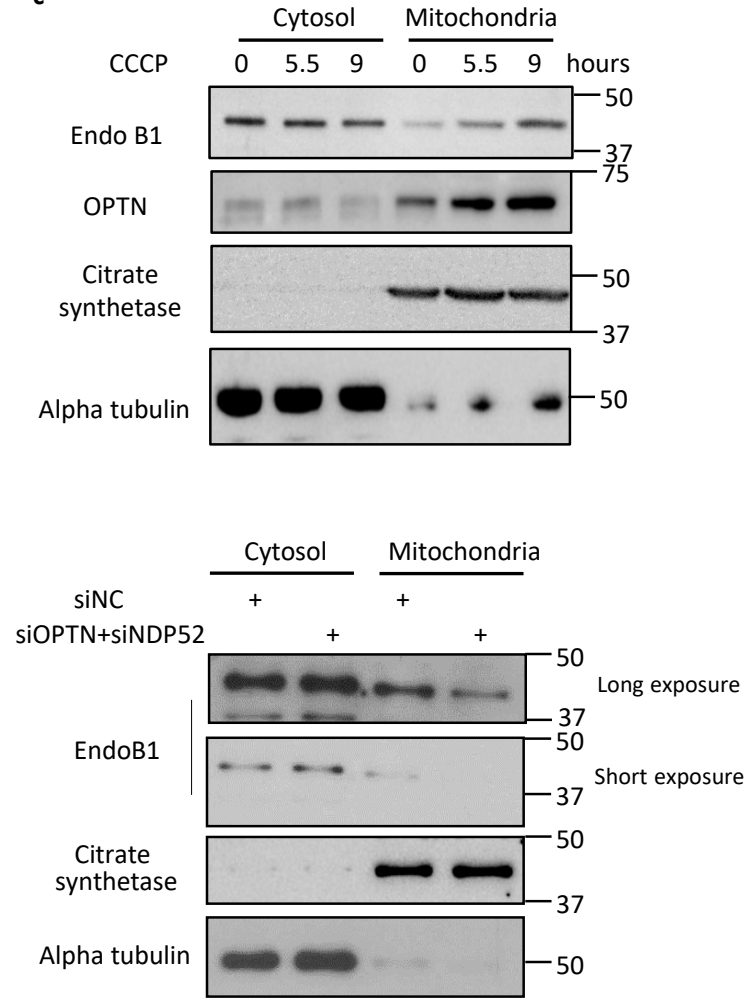

g

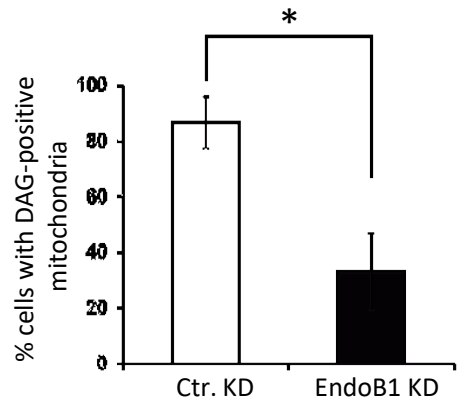

Fig. 6 OPTN and NDP52 deliver EndoB1-positive golgi vesicles to ubiquitinated mitochondria for mitochondrial DAG production. a,b Hela cells were co-transfected with expression plasmids for GFP-TGN38, mCherry-OPTN and FLAG-Parkin a or mCherry-OPTN and GFP-Parkin b and treated with CCCP for $5.5 \mathrm{hrs}$. Mitochondria were visualized by immunostaining with a Tom20 antibody and EndoB1 localization was assessed by an EndoB1 antibody. Note that GFP-TGN38 (a bottom panel) and EndoB1 (b bottom panel), pseudo colored in white in single channel and blue in the overlay images, translocates to mitochondria. c Mitochondrial and cytosolic fractions obtained from control and CCCP treated cells, as was described and analyzed in Fig. 2b, were immunoblotted for EndoB1 and OPTN, as indicated. d Hela OPTN/NDP52 knockdown cells were transfected with YFP-DAGR and mCherry-Parkin and treated with CCCP as indicated. EndoB1 localization was assessed by an EndoB1 antibody and was presented in pseudo color white as $\mathbf{b}$. Note that translocation of EndoB1 to mitochondria was impaired in OPTN/NDP52 knockdown cells (bottom panel). e Cytosolic and mitochondrial fractions purified from control and OPTN/NDP52 knockdown cells treated with CCCP were immunoblotted for antibodies, as indicated. Note that mitochondrial EndoB1 levels were reduced in OPTN/NDP52 knockdown cells. f,g Hela cells were transfected with an EndoB1 siRNA, followed by transfection of expression plasmids for mCherry-Parkin and YFP-DAGR, and CCCP treatment at $10 \mu \mathrm{M}$ for $9 \mathrm{hrs}$. Cells with mitochondrial YFP-DAGR were quantified from $\mathbf{f}\left({ }^{*} \mathrm{P}<0.05\right.$, Student's t-test). Scale bar $=25 \mu \mathrm{M}$ and zoom is $5 \mathrm{x}$. Note that knockdown of EndoB1 suppressed mitochondrial DAG production. 